\title{
LIE ALGEBRAS GENERATED BY LIE MODULES
}

\author{
Nagatoshi SASANO \\ (Received 6 October 2013)
}

\begin{abstract}
It is known that a finite-dimensional reductive Lie algebra has a non-degenerate symmetric invariant bilinear form. In this paper, for a given reductive Lie algebra and its finite-dimensional completely reducible representation, we will construct a graded Lie algebra by using a non-degenerate symmetric invariant bilinear form on the reductive Lie algebra. This graded Lie algebra also has a non-degenerate symmetric invariant bilinear form and, moreover, the reductive Lie algebra, its representation and the bilinear form which are used to construct the graded Lie algebra can be embedded into it.
\end{abstract}

\section{Introduction}

Let $G$ be a connected algebraic group, $\rho$ a representation of $G$ on a finite-dimensional vector space $V$, all defined over $\mathbb{C}$. When there exists an element $v \in V$ such that the orbit $\rho(G) v$ is Zariski dense in $V$, we say that the triplet $(G, \rho, V)$ is a prehomogeneous vector space (PV) and $v$ is called a generic point (see $[\mathbf{S}-\mathbf{K}]$ ). The theory of PVs is closely related to the theory of Lie algebras. We can express the prehomogeneity condition of a triplet $(G, \rho, V)$ by the method of Lie algebras as follows.

We denote the Lie algebra of $G$ by $\operatorname{Lie}(G)$ and the infinitesimal representation of $\rho$ by $d \rho$. Then $(G, \rho, V)$ is a PV if and only if there exists an element $v \in V$ which satisfies $d \rho(\operatorname{Lie}(G)) v=V$. Such an element $v$ is a generic point. This is an infinitesimal condition of the prehomogeneity (see [S-K, Proposition 2, p. 36]). Moreover, we have a class of PVs called PVs of parabolic type. These PVs can be obtained by graded semisimple Lie algebras in the following way.

Let $\mathfrak{g}$ be a finite-dimensional semisimple Lie algebra, $G$ the adjoint group of $\mathfrak{g}, \mathfrak{h}$ a Cartan subalgebra of $\mathfrak{g}, R$ the root system of $\mathfrak{g}$ with respect to $\mathfrak{h}, \varphi$ a fundamental system of $R$ all defined over $\mathbb{C}$. Let $\theta$ be a subset of $\varphi$ and $H^{\theta} \in \mathfrak{h}$ the unique element which satisfies $\alpha\left(H^{\theta}\right)=0$ for all $\alpha \in \theta$ and $\alpha\left(H^{\theta}\right)=2$ for all $\alpha \in \varphi \backslash \theta$. Put $d_{n}(\theta):=\left\{X \in \mathfrak{g} \mid\left[H^{\theta}, X\right]=\right.$ $2 n X\}$ for each $n \in \mathbb{Z}$. Then we can obtain a $\mathbb{Z}$-grading of $\mathfrak{g}$ :

$$
\mathfrak{g}=\bigoplus_{n \in \mathbb{Z}} d_{n}(\theta)
$$

The graded Lie algebra (0.1) and the Killing form $K$ of $\mathfrak{g}$ satisfy the following conditions:

$d_{0}(\theta)$ and $d_{1}(\theta)$ are finite-dimensional vector spaces;

the adjoint representation of $d_{0}(\theta)$ on $d_{1}(\theta)$ is completely reducible;

the restriction of $K$ to $d_{i}(\theta) \times d_{-i}(\theta)$ is non-degenerate for each $i \geq 0$;

$$
\left[d_{1}(\theta), d_{i}(\theta)\right]=d_{i+1}(\theta),\left[d_{-1}(\theta), d_{-j}(\theta)\right]=d_{-j-1}(\theta) \text { for each } i, j \geq 0 .
$$

2010 Mathematics Subject Classification: Primary 17B70, 11S90; Secondary 17B65.

Keywords: graded Lie algebras; prehomogeneous vector spaces of parabolic type.

(C) 2014 Faculty of Mathematics, Kyushu University 
Denote $d_{0}(\theta)$ by $\mathfrak{l}_{\theta}$ and the connected subgroup of $G$ which corresponds to $\mathfrak{l}_{\theta}$ by $L_{\theta}$, then it is known that $\left(L_{\theta}, d_{n}(\theta)\right)$ is a PV for each $n \neq 0$ denoted by $\left(\mathfrak{l}_{\theta}, d_{n}(\theta)\right)$, i.e. there exists an element $v \in d_{n}(\theta)$ such that $\left[\mathfrak{l}_{\theta}, v\right]=d_{n}(\theta)$. Rubenthaler [Ru-1, Ru-2, Ru-3], studied spaces of the form $\left(\mathfrak{l}_{\theta}, d_{1}(\theta)\right)$ and called them PV of parabolic type. These spaces can be described and classified by the weighted Dynkin diagrams. Moreover, we can recognize the space $\left(\mathfrak{l}_{\theta}, d_{-1}(\theta)\right)$ as the dual space of $\left(\mathfrak{l}_{\theta}, d_{1}(\theta)\right)$ via the Killing form $K$ of $\mathfrak{g}$ and assume that $\left[d_{1}(\theta), d_{-1}(\theta)\right]=\mathfrak{l}_{\theta}$ without loss of generality, i.e. we can say that a PV of parabolic type is a triplet which consists of a reductive Lie algebra and its representation which can be embedded into a finite-dimensional graded Lie algebra with a non-degenerate symmetric invariant bilinear form.

The purpose of this paper is to introduce a way to embed a given finite-dimensional reductive Lie algebra $\mathfrak{g}$, a finite-dimensional completely reducible representation $(\rho, V)$ (do not assume the prehomogeneity condition) of $\mathfrak{g}$ and its dual space $\left(\rho^{*}, V^{*}\right)$ into a 'large' graded Lie algebra with a non-degenerate symmetric invariant bilinear form. In particular, a PVs of parabolic type can be embedded into a finite-dimensional semisimple Lie algebra by our construction. For this, we use a non-degenerate symmetric invariant bilinear form $B_{0}$ on $\mathfrak{g}$. That is, for a standard quadruplet $\left(\mathfrak{g}, \rho, V, B_{0}\right.$ ) (Definition 1.9), we construct a graded Lie algebra of the form $L\left(\mathfrak{g}, \rho, V, B_{0}\right)=\bigoplus_{n \in \mathbb{Z}} V_{n}$ with a non-degenerate symmetric invariant bilinear form $B$ such that $V_{0}=\mathfrak{g}, V_{1}$ is isomorphic to $V$ as a $\mathfrak{g}$-module and $B_{0}$ coincides with a restriction of $B$ to $V_{0} \times V_{0}$ (Theorem 2.11 and Proposition 3.2). Moreover, we prove that $L\left(\mathfrak{g}, \rho, V, B_{0}\right)$ satisfies the following conditions:

$V_{0}$ and $V_{1}$ are finite-dimensional vector spaces;

the adjoint representation of $V_{0}$ on $V_{1}$ is completely reducible;

the restriction of $B$ to $V_{i} \times V_{-i}$ is non-degenerate for each $i \geq 0$;

$\left[V_{1}, V_{i}\right]=V_{i+1},\left[V_{-1}, V_{-j}\right]=V_{-j-1}$ for each $i, j \geq 0$;

$\left[V_{1}, V_{-1}\right]=V_{0}$.

Conversely, an arbitrary graded Lie algebra which satisfies the conditions from (0.6) to (0.10) can be obtained by our construction (Proposition 3.3). In particular, an arbitrary finitedimensional semisimple Lie algebra can be obtained by a certain PV of parabolic type and a bilinear form (Theorem 3.13).

This paper consists of the following three sections.

In Section 1, first of all, we will explain that a quadruplet $\left(\mathfrak{g}, \rho, V, B_{0}\right)$ determines a linear map $\hat{\Phi}$ from $V \otimes V^{*}$ to $\mathfrak{g}$. Then $\hat{\Phi}$ induces a $\mathfrak{g}$-submodule $V_{1}$ of $\operatorname{Hom}\left(V^{*}, \mathfrak{g}\right)$. In order to have $V_{1} \simeq V$ as $\mathfrak{g}$-modules, we introduce the notion of standard quadruplets. Then, moreover, we can obtain $\mathfrak{g}$-modules $V_{n}$ for all $n \in \mathbb{Z}$ inductively. We call $V_{n}$ the $n$-graduation of $\left(\mathfrak{g}, \rho, V, B_{0}\right)$. Each $\mathfrak{g}$-module $V_{n}$ is finite-dimensional and the dual $\mathfrak{g}$-module of $V_{-n}$. In particular, $V_{0}$ is isomorphic to $\mathfrak{g}$ itself.

In Section 2, we define a bilinear map [·, $\cdot]_{m}^{n}$ from $V_{n} \times V_{m}$ to $V_{n+m}$ for any $n, m \in \mathbb{Z}$. Denote the direct sum of $n$-graduations obtained in Section 1 by $L\left(\mathfrak{g}, \rho, V, B_{0}\right)$. Then we have a bilinear map $[\cdot, \cdot]: L\left(\mathfrak{g}, \rho, V, B_{0}\right) \times L\left(\mathfrak{g}, \rho, V, B_{0}\right) \rightarrow L\left(\mathfrak{g}, \rho, V, B_{0}\right)$ defined by

$$
\left[x_{n}, y_{m}\right]:=\left[x_{n}, y_{m}\right]_{m}^{n} \quad\left(x_{n} \in V_{n}, y_{m} \in V_{m}\right) .
$$


Our main result is that $[\cdot, \cdot]$ satisfies the axioms of a Lie algebra, i.e. $L\left(\mathfrak{g}, \rho, V, B_{0}\right)$ has a structure of a graded Lie algebra. We call the Lie algebra thus obtained a Lie algebra associated with a standard quadruplet (Theorem 2.11). For example, loop algebras and finite-dimensional semisimple Lie algebras are Lie algebras associated with some standard quadruplet. This will be proved in Section 3.

In Section 3, we will study some properties of $L\left(\mathfrak{g}, \rho, V, B_{0}\right)$. First, we construct a nondegenerate symmetric and invariant bilinear form on $L\left(\mathfrak{g}, \rho, V, B_{0}\right)$. Then we can prove that the Lie algebras associated with a standard quadruplet can be characterized by the existence of a non-degenerate symmetric and invariant bilinear form. This is described in Proposition 3.3. Finally, in Theorem 3.13, we will give a necessary and sufficient condition for $\left(\mathfrak{g}, \rho, V, B_{0}\right)$ to generate a finite-dimensional Lie algebra $L\left(\mathfrak{g}, \rho, V, B_{0}\right)$. The condition is related to PVs of parabolic type.

Throughout this paper, we shall always assume that all objects are defined over the complex number field $\mathbb{C}$.

\section{A family of $\boldsymbol{n}$-graduations}

\subsection{Standard quadruplets}

Let $\mathfrak{g}$ be a finite-dimensional reductive Lie algebra, $V$ a finite-dimensional vector space and $\rho$ a representation of $\mathfrak{g}$ on $V$ all defined over $\mathbb{C}$. By the theory of Lie algebras, there exists a non-degenerate symmetric $\mathfrak{g}$-invariant bilinear form $B_{0}$ on $\mathfrak{g}$ (See $[\mathbf{B u}$, Chapter $1, \S 6.4$, Proposition 5]). Let $V^{*}$ be the dual module of $V$ and $\rho^{*}$ the dual representation of $\rho$. We denote the pairing between $V$ and $V^{*}$ by $\langle\cdot, \cdot\rangle$. By the assumption that $B_{0}$ is non-degenerate, we can define the following linear maps.

Definition 1.1. We define a linear map $\hat{\Phi}$ (respectively $\hat{\Psi}$ ) from $V \otimes V^{*}$ to $\mathfrak{g}$ (respectively from $V^{*} \otimes V$ to $\mathfrak{g}$ ) by the following equation:

$$
B_{0}(a, \hat{\Phi}(v \otimes \phi))=\langle\rho(a) v, \phi\rangle=-\left\langle v, \rho^{*}(a) \phi\right\rangle
$$

respectively

$$
B_{0}(a, \hat{\Psi}(\phi \otimes v))=\left\langle v, \rho^{*}(a) \phi\right\rangle=-\langle\rho(a) v, \phi\rangle
$$

for any $a \in \mathfrak{g}, v \in V$ and $\phi \in V^{*}$. We call this map $\hat{\Phi}$ (respectively $\hat{\Psi}$ ) the $\Phi$-map (respectively the $\Psi$-map) of the quadruplet ( $\left.\mathfrak{g}, \rho, V, B_{0}\right)$.

Remark 1.2. For any $v \in V$ and $\phi \in V^{*}$, we have

$$
\hat{\Phi}(v \otimes \phi)+\hat{\Psi}(\phi \otimes v)=0 .
$$

Here, $V \otimes V^{*}$ and $V^{*} \otimes V$ have canonical $\mathfrak{g}$-module structures and we have the following proposition.

Proposition 1.3. The maps $\hat{\Phi}$ and $\hat{\Psi}$ are homomorphisms of $\mathfrak{g}$-modules. 
Proof. In fact, for any $a, a^{\prime} \in \mathfrak{g}, v \in V$ and $\phi \in V^{*}$, we have

$$
\begin{aligned}
B_{0} & \left(a^{\prime}, \hat{\Phi}\left((\rho(a) v) \otimes \phi+v \otimes\left(\rho^{*}(a) \phi\right)\right)\right) \\
& =\left\langle\rho\left(a^{\prime}\right) \rho(a) v, \phi\right\rangle+\left\langle\rho\left(a^{\prime}\right) v, \rho^{*}(a) \phi\right\rangle \\
& =\left\langle\rho\left(\left[a^{\prime}, a\right]\right) v, \phi\right\rangle+\left\langle\rho(a) \rho\left(a^{\prime}\right) v, \phi\right\rangle+\left\langle\rho\left(a^{\prime}\right) v, \rho^{*}(a) \phi\right\rangle \\
& =B_{0}\left(\left[a^{\prime}, a\right], \hat{\Phi}(v \otimes \phi)\right)-\left\langle\rho\left(a^{\prime}\right) v, \rho^{*}(a) \phi\right\rangle+\left\langle\rho\left(a^{\prime}\right) v, \rho^{*}(a) \phi\right\rangle \\
& =B_{0}\left(a^{\prime},[a, \hat{\Phi}(v \otimes \phi)]\right) .
\end{aligned}
$$

Hence, we have $\hat{\Phi}\left((\rho(a) v) \otimes \phi+v \otimes\left(\rho^{*}(a) \phi\right)\right)=[a, \hat{\Phi}(v \otimes \phi)]$. Similarly, we can obtain the equation $\hat{\Psi}\left(\left(\rho^{*}(a) \phi\right) \otimes v+\phi \otimes(\rho(a) v)\right)=[a, \hat{\Psi}(\phi \otimes v)]$ and thus $\hat{\Phi}$ and $\hat{\Psi}$ are homomorphisms of $\mathfrak{g}$-modules.

By Proposition 1.3, $\hat{\Phi}\left(V \otimes V^{*}\right)$ and $\hat{\Phi}\left(V \otimes V^{*}\right)^{\perp}:=\left\{a \in \mathfrak{g} \mid B_{0}(a, x)=0\right.$ for any $x \in$ $\left.\hat{\Phi}\left(V \otimes V^{*}\right)\right\}$ are ideals of $\mathfrak{g}$. Then $\hat{\Phi}\left(V \otimes V^{*}\right)^{\perp}$ coincides with Ker $\rho$. In fact, suppose that $a \in \operatorname{Ker} \rho$. Then we have $B_{0}(a, \hat{\Phi}(v \otimes \phi))=\langle\rho(a) v, \phi\rangle=0$ for any $v \in V$ and $\phi \in V^{*}$. Thus, we have $\hat{\Phi}\left(V \otimes V^{*}\right)^{\perp} \supset \operatorname{Ker} \rho$. Similarly we have the converse inclusion. Hence, we obtain the following proposition.

Proposition 1.4. The maps $\hat{\Phi}$ and $\hat{\Psi}$ are surjective if and only if $\rho$ is faithful.

Next, let us construct $\mathfrak{g}$-modules which are isomorphic to $V$ and $V^{*}$, respectively.

Definition 1.5. For each element $v \in V$ and $\phi \in V^{*}$, we define linear maps $\Phi_{v} \in \operatorname{Hom}\left(V^{*}, \mathfrak{g}\right)$ and $\Psi_{\phi} \in \operatorname{Hom}(V, \mathfrak{g})$ by

$$
\begin{aligned}
\Phi_{v}(\psi) & :=\hat{\Phi}(v \otimes \psi), \\
\Psi_{\phi}(u) & :=\hat{\Psi}(\phi \otimes u),
\end{aligned}
$$

where $u \in V$ and $\psi \in V^{*}$. Then we have linear maps $\Phi$ and $\Psi$ as follows:

$$
\begin{aligned}
\Phi: V & \rightarrow \operatorname{Hom}\left(V^{*}, \mathfrak{g}\right) \\
v & \mapsto \Phi_{v}, \\
\Psi: V^{*} & \rightarrow \operatorname{Hom}(V, \mathfrak{g}) \\
\phi & \mapsto \Psi_{\phi} .
\end{aligned}
$$

The spaces $\operatorname{Hom}(\mathfrak{g}, V)$ and $\operatorname{Hom}\left(\mathfrak{g}, V^{*}\right)$ have canonical $\mathfrak{g}$-module structures. Then we have the following proposition.

PROPOSITION 1.6. The maps $\Phi$ and $\Psi$ are homomorphisms of $\mathfrak{g}$-modules.

Proof. By Proposition 1.3, we have the following equations:

$$
\begin{aligned}
\Phi_{\rho(a) v}(\phi) & =\left[a, \Phi_{v}(\phi)\right]-\Phi_{v}\left(\rho^{*}(a) \phi\right), \\
\Psi_{\rho^{*}(b) \psi}(u) & =\left[b, \Psi_{\psi}(u)\right]-\Psi_{\psi}(\rho(b) u)
\end{aligned}
$$

for any $a, b \in \mathfrak{g}, v, u \in V$ and $\phi, \psi \in V^{*}$. Thus, we have our result. 
Put

$$
\begin{aligned}
V_{0} & :=\mathfrak{g}, \\
V_{1} & :=\operatorname{Im} \Phi, \\
V_{-1} & :=\operatorname{Im} \Psi .
\end{aligned}
$$

Then $V_{0}, V_{1}$ and $V_{-1}$ are $\mathfrak{g}$-modules. We denote the canonical representations of $\mathfrak{g}$ on $V_{0}, V_{1}$ and $V_{-1}$ by $\rho_{0}, \rho_{1}$ and $\rho_{-1}$, respectively. Then we can define linear maps $p_{0}$ and $q_{0}$ by

$$
\begin{aligned}
p_{0}: V_{1} \otimes V_{0} & \rightarrow V_{1} \\
v_{1} \otimes a & \mapsto-\rho_{1}(a) v_{1}, \\
q_{0}: V_{-1} \otimes V_{0} & \rightarrow V_{-1} \\
\phi_{-1} \otimes a & \mapsto-\rho_{-1}(a) \phi_{-1} .
\end{aligned}
$$

We can easily show that $p_{0}$ and $q_{0}$ are homomorphisms of $\mathfrak{g}$-modules. Furthermore, if $\rho$ is completely reducible and does not have a subrepresentation on a non-zero subspace of $V$ which is isomorphic to the zero representation, then $p_{0}$ and $q_{0}$ are surjective and $\Phi$ and $\Psi$ are injective. In fact, take an element $v \in V$ which satisfies $\Phi_{v}=0$. Then we have $\langle\rho(a) v, \phi\rangle=B_{0}\left(a, \Phi_{v}(\phi)\right)=0$ for any $a \in \mathfrak{g}$ and $\phi \in V^{*}$, hence $\rho(a) v=0$ and $v=0$. The same holds for $\Psi$. Therefore, we obtain the following proposition.

PROPOSITION 1.7. If $\rho$ is completely reducible and does not have a subrepresentation on a non-zero subspace of $V$ which is isomorphic to the zero representation, then $\mathfrak{g}$-modules $V_{1}$ and $V_{-1}$ are isomorphic to $V$ and $V^{*}$, respectively.

Remark 1.8. In the situation of Proposition 1.7, we have

$$
v_{1}\left(\Psi^{-1}\left(\phi_{-1}\right)\right)+\phi_{-1}\left(\Phi^{-1}\left(v_{1}\right)\right)=0
$$

for any $v_{1} \in V_{1}$ and $\phi_{-1} \in V_{-1}$.

Here, we give the following definition.

Definition 1.9. Let $\mathfrak{g}$ be a finite-dimensional reductive Lie algebra, $V$ a finite-dimensional vector space, $\rho$ a representation of $\mathfrak{g}$ on $V, B_{0}$ a non-degenerate symmetric invariant bilinear form on $\mathfrak{g}$. If a quadruplet $\left(\mathfrak{g}, \rho, V, B_{0}\right)$ satisfies the following conditions, we call it a standard quadruplet:

$\rho$ is completely reducible,

$\rho$ is faithful,

$\rho$ does not have a subrepresentation on a non-zero subspace of $V$ which is isomorphic to the zero-representation.

In particular, if $\left(\mathfrak{g}, \rho, V, B_{0}\right)$ is a standard quadruplet, the $\mathfrak{g}$-modules $V_{0}, V_{1}$ and $V_{-1}$ are isomorphic to $\hat{\Phi}\left(V \otimes V^{*}\right), V$ and $V^{*}$, respectively. Next, let us construct $\mathfrak{g}$-modules from $V_{1}$ and $V_{-1}$. For this, we shall show the following proposition used later. 
Proposition 1.10. Let $\left(\mathfrak{g}, \rho, V, B_{0}\right)$ be a standard quadruplet. Let $W$ be a finitedimensional $\mathfrak{g}$-module and $\rho_{W}$ a representation of $\mathfrak{g}$ on $W$. Assume that there exists a homomorphism of $\mathfrak{g}$-modules $p$ from $V_{1} \otimes W$ to $\operatorname{Hom}\left(V^{*}, W\right)$. We put

$$
\tilde{W}:=\operatorname{Im} p
$$

and denote the canonical representation of $\mathfrak{g}$ on $\tilde{W}$ by $\tilde{\rho}_{W}$. Then the following linear map $\tilde{p}$ defined by

$$
\begin{aligned}
\tilde{p}: V_{1} \otimes \tilde{W} & \rightarrow \operatorname{Hom}\left(V^{*}, \tilde{W}\right) \\
v_{1} \otimes \tilde{w} & \mapsto\left(\phi \mapsto \tilde{\rho}_{W}\left(v_{1}(\phi)\right) \tilde{w}+p\left(v_{1} \otimes \tilde{w}(\phi)\right)\right)
\end{aligned}
$$

is a homomorphism of $\mathfrak{g}$-modules.

Proof. For any $a \in \mathfrak{g}, v_{1} \in V_{1}, \tilde{w} \in \tilde{W}$ and $\phi \in V^{*}$, we have

$$
\begin{aligned}
& \tilde{p}\left(\rho_{1}(a) v_{1} \otimes \tilde{w}\right)(\phi)+\tilde{p}\left(v_{1} \otimes \tilde{\rho}_{W}(a) \tilde{w}\right)(\phi) \\
&= \tilde{\rho}_{W}\left(\left(\rho_{1}(a) v_{1}\right)(\phi)\right) \tilde{w}+p\left(\rho_{1}(a) v_{1} \otimes \tilde{w}(\phi)\right) \\
&+\tilde{\rho}_{W}\left(v_{1}(\phi)\right) \tilde{\rho}_{W}(a) \tilde{w}+p\left(v_{1} \otimes\left(\tilde{\rho}_{W}(a) \tilde{w}\right)(\phi)\right) \\
&= \tilde{\rho}_{W}\left(\left[a, v_{1}(\phi)\right]\right) \tilde{w}-\tilde{\rho}_{W}\left(v_{1}\left(\rho^{*}(a) \phi\right)\right) \tilde{w} \\
&+p\left(\rho_{1}(a) v_{1} \otimes \tilde{w}(\phi)\right)+\tilde{\rho}_{W}\left(v_{1}(\phi)\right) \tilde{\rho}_{W}(a) \tilde{w} \\
&+p\left(v_{1} \otimes \rho_{W}(a)(\tilde{w}(\phi))\right)-p\left(v_{1} \otimes \tilde{w}\left(\rho^{*}(a) \phi\right)\right) \\
&= \tilde{\rho}_{W}(a) \tilde{\rho}_{W}\left(v_{1}(\phi)\right) \tilde{w}+\tilde{\rho}_{W}(a) p\left(v_{1} \otimes(\tilde{w}(\phi))\right) \\
&-\tilde{p}\left(v_{1} \otimes \tilde{w}\right)\left(\rho^{*}(a) \phi\right) \\
&= \tilde{\rho}_{W}(a)\left(\tilde{p}\left(v_{1} \otimes \tilde{w}\right)(\phi)\right)-\tilde{p}\left(v_{1} \otimes \tilde{w}\right)\left(\rho^{*}(a) \phi\right) .
\end{aligned}
$$

Thus, we have our result.

Definition 1.11. Let $\left(\mathfrak{g}, \rho, V, B_{0}\right)$ be a standard quadruplet. Suppose that $i \geq 1$ and there exist $\mathfrak{g}$-modules $\left(\rho_{i-1}, V_{i-1}\right)$ and $\left(\rho_{-i+1}, V_{-i+1}\right)$ and homomorphisms of $\mathfrak{g}$-modules $p_{i-1}$ : $V_{1} \otimes V_{i-1} \rightarrow \operatorname{Hom}\left(V^{*}, V_{i-1}\right)$ and $q_{-i+1}: V_{-1} \otimes V_{-i+1} \rightarrow \operatorname{Hom}\left(V, V_{-i+1}\right)$. Put $V_{i}:=$ $\operatorname{Im} p_{i-1}$ and $V_{-i}:=\operatorname{Im} q_{-i+1}$. (Note that $p_{0}$ and $q_{0}$ defined in (1.13) and (1.14) are surjective.) We define linear maps $p_{i}$ and $q_{-i}$ by

$$
\begin{aligned}
p_{i}: & V_{1} \otimes V_{i} \rightarrow \operatorname{Hom}\left(V^{*}, V_{i}\right) \\
v_{1} \otimes u_{i} & \mapsto\left(\phi \mapsto \rho_{i}\left(v_{1}(\phi)\right) u_{i}+p_{i-1}\left(v_{1} \otimes u_{i}(\phi)\right)\right), \\
q_{-i}: & V_{-1} \otimes V_{-i} \rightarrow \operatorname{Hom}\left(V, V_{-i}\right) \\
\phi_{-1} \otimes \psi_{-i} & \mapsto\left(v \mapsto \rho_{-i}\left(\phi_{-1}(v)\right) \psi_{-i}+q_{-i+1}\left(\phi_{-1} \otimes \psi_{-i}(v)\right)\right) .
\end{aligned}
$$

Then, by Proposition 1.10, $p_{i}$ and $q_{-i}$ are homomorphisms of $\mathfrak{g}$-modules. We denote the images of $p_{i}$ and $q_{-i}$ by $V_{i+1}$ and $V_{-i-1}$ and the canonical representations on $V_{i+1}$ and $V_{-i-1}$ by $\rho_{i+1}$ and $\rho_{-i-1}$, respectively. Thus, inductively, we obtain $\mathfrak{g}$-modules $V_{n}$ and representations $\rho_{n}$ of $\mathfrak{g}$ on $V_{n}$ for all $n \in \mathbb{Z}$. We call $V_{n}$ the $n$-graduation of $\left(\mathfrak{g}, \rho, V, B_{0}\right)$. Throughout this paper, we use these notation. 
Remark 1.12. In particular, if $i=1$, we have

$$
\begin{aligned}
p_{1}\left(v_{1} \otimes u_{1}\right)(\phi) & =-p_{1}\left(u_{1} \otimes v_{1}\right)(\phi) \\
& =\rho_{1}\left(v_{1}(\phi)\right) u_{1}-\rho_{1}\left(u_{1}(\phi)\right) v_{1}
\end{aligned}
$$

and

$$
\begin{aligned}
q_{-1}\left(\phi_{-1} \otimes \psi_{-1}\right)(v) & =-q_{-1}\left(\psi_{-1} \otimes \phi_{-1}\right)(v) \\
& =\rho_{-1}\left(\phi_{-1}(v)\right) \psi_{-1}-\rho_{-1}\left(\psi_{-1}(v)\right) \phi_{-1}
\end{aligned}
$$

under the above notation.

\subsection{A bilinear form on $V_{i} \times V_{-i}$}

In this section, let us consider the relation between $\mathfrak{g}$-modules $V_{i}$ and $V_{-i}$ for each $i \geq 0$. For this, we shall construct a bilinear form on $V_{i} \times V_{-i}$. First, let us show that $B_{0}$ satisfies the following equation:

$$
B_{0}\left(p_{0}\left(v_{1} \otimes a\right)\left(\Psi^{-1}\left(\phi_{-1}\right)\right), b\right)=B_{0}\left(a, q_{0}\left(\phi_{-1} \otimes b\right)\left(\Phi^{-1}\left(v_{1}\right)\right)\right),
$$

where $a, b \in V_{0}, v_{1} \in V_{1}$ and $\phi_{-1} \in V_{-1}$.

Put $v:=\Phi^{-1}\left(v_{1}\right)$ and $\phi:=\Psi^{-1}\left(\phi_{-1}\right)$. Then we have

$$
\begin{aligned}
B_{0} & \left(p_{0}\left(v_{1} \otimes a\right)(\phi), b\right) \\
& =B_{0}\left(\left(-\rho_{1}(a) v_{1}\right)(\phi), b\right) \\
& =B_{0}\left(v_{1}\left(\rho^{*}(a) \phi\right), b\right)-B_{0}\left(\left[a, v_{1}(\phi)\right], b\right) \\
& =\left\langle\rho(b) v, \rho^{*}(a) \phi\right\rangle+B_{0}\left(\left[a, \phi_{-1}(v)\right], b\right) \\
& =B_{0}\left(a, \phi_{-1}(\rho(b) v)\right)+B_{0}\left(a,\left[\phi_{-1}(v), b\right]\right) \\
& =B_{0}\left(a, q_{0}\left(\phi_{-1} \otimes b\right)(v)\right) .
\end{aligned}
$$

Next, suppose that $i \geq 1$. Assume that there exists a $\mathfrak{g}$-invariant bilinear form $B_{i-1}$ on $V_{i-1} \times V_{-i+1}$ which satisfies the following equation:

$$
\begin{aligned}
& B_{i-1}\left(p_{i-1}\left(v_{1} \otimes u_{i-1}\right)\left(\Psi^{-1}\left(\phi_{-1}\right)\right), \psi_{-i+1}\right) \\
& \quad=B_{i-1}\left(u_{i-1}, q_{-i+1}\left(\phi_{-1} \otimes \psi_{-i+1}\right)\left(\Phi^{-1}\left(v_{1}\right)\right)\right)
\end{aligned}
$$

for any $v_{1} \in V_{1}, u_{i-1} \in V_{i-1}, \phi_{-1} \in V_{-1}$ and $\psi_{-i+1} \in V_{-i+1}$. Then we can define a bilinear form $B_{i}$ on $V_{i} \times V_{-i}$ by

$$
\begin{aligned}
& B_{i}\left(p_{i-1}\left(v_{1} \otimes u_{i-1}\right), q_{-i+1}\left(\phi_{-1} \otimes \psi_{-i+1}\right)\right) \\
& \quad:=B_{i-1}\left(p_{i-1}\left(v_{1} \otimes u_{i-1}\right)\left(\Psi^{-1}\left(\phi_{-1}\right)\right), \psi_{-i+1}\right),
\end{aligned}
$$

where $v_{1} \in V_{1}, u_{i-1} \in V_{i-1}, \phi_{-1} \in V_{-1}$ and $\psi_{-i+1} \in V_{-i+1}$. Indeed, by (1.26), $B_{i}$ is well defined. Then $B_{i}$ is $\mathfrak{g}$-invariant. In fact, by the assumption that $B_{i-1}$ is $\mathfrak{g}$-invariant, we have

$$
\begin{aligned}
& B_{i}\left(\rho_{i}(a) p_{i-1}\left(v_{1} \otimes u_{i-1}\right), q_{-i+1}\left(\phi_{-1} \otimes \psi_{-i+1}\right)\right) \\
&= B_{i-1}\left(\rho_{i-1}(a)\left(p_{i-1}\left(v_{1} \otimes u_{i-1}\right)\left(\Psi^{-1}\left(\phi_{-1}\right)\right)\right), \psi_{-i+1}\right) \\
&-B_{i-1}\left(p_{i-1}\left(v_{1} \otimes u_{i-1}\right)\left(\rho^{*}(a) \Psi^{-1}\left(\phi_{-1}\right)\right), \psi_{-i+1}\right)
\end{aligned}
$$




$$
\begin{aligned}
= & -B_{i-1}\left(p_{i-1}\left(v_{1} \otimes u_{i-1}\right)\left(\Psi^{-1}\left(\phi_{-1}\right)\right), \rho_{-i+1}(a) \psi_{-i+1}\right) \\
& -B_{i-1}\left(p_{i-1}\left(v_{1} \otimes u_{i-1}\right)\left(\Psi^{-1}\left(\rho_{-1}(a) \phi_{-1}\right)\right), \psi_{-i+1}\right) \\
= & -B_{i}\left(p_{i-1}\left(v_{1} \otimes u_{i-1}\right), \rho_{-i}(a) q_{-i+1}\left(\phi_{-1} \otimes \psi_{-i+1}\right)\right)
\end{aligned}
$$

for any $a \in \mathfrak{g}, v_{1} \in V_{1}, u_{i-1} \in V_{i-1}, \phi_{-1} \in V_{-1}$ and $\psi_{-i+1} \in V_{-i+1}$. Moreover, $B_{i}$ satisfies the following equation:

$$
B_{i}\left(p_{i}\left(v_{1} \otimes u_{i}\right)\left(\Psi^{-1}\left(\phi_{-1}\right)\right), \psi_{-i}\right)=B_{i}\left(u_{i}, q_{-i}\left(\phi_{-1} \otimes \psi_{-i}\right)\left(\Phi^{-1}\left(v_{1}\right)\right)\right)
$$

for any $v_{1} \in V_{1}, u_{i} \in V_{i}, \phi_{-1} \in V_{-1}$ and $\psi_{-i} \in V_{-i}$. In fact, put $v:=\Phi^{-1}\left(v_{1}\right)$ and $\phi:=$ $\Psi^{-1}\left(\phi_{-1}\right)$. Then we have

$$
\begin{aligned}
B_{i} & \left(p_{i}\left(v_{1} \otimes u_{i}\right)\left(\Psi^{-1}\left(\phi_{-1}\right)\right), \psi_{-i}\right) \\
& =B_{i}\left(\rho_{i}\left(v_{1}(\phi)\right) u_{i}+p_{-1}\left(v_{1} \otimes u_{i}(\phi)\right), \psi_{-i}\right) \\
& =B_{i}\left(u_{i}, \rho_{-i}\left(\phi_{-1}(v)\right) \psi_{-i}\right)+B_{i-1}\left(u_{i}(\phi), \psi_{-i}(v)\right) \\
& =B_{i}\left(u_{i}, \rho_{-i}\left(\phi_{-1}(v)\right) \psi_{-i}\right)+B_{i}\left(u_{i}, q_{-i}\left(\phi_{-1} \otimes \psi_{-i}(v)\right)\right) \\
& =B_{i}\left(u_{i}, q_{-i}\left(\phi_{-1} \otimes \psi_{-i}\right)\left(\Phi^{-1}\left(v_{1}\right)\right)\right) .
\end{aligned}
$$

Thus, inductively, we obtain a bilinear form $B_{i}$ on $V_{i} \times V_{-i}$ for all $i \geq 0$.

Proposition 1.13. For all $i \geq 0, V_{-i}$ is the dual $\mathfrak{g}$-module of $V_{i}$.

Proof. It is sufficient to show that the bilinear map $B_{i}: V_{i} \times V_{-i} \rightarrow \mathbb{C}$ is non-degenerate for all $i \geq 0$. We argue by induction on $i$. For $i=0$, by the assumption, $B_{0}: V_{0} \times V_{0} \rightarrow \mathbb{C}$ is non-degenerate and $\mathfrak{g}$-invariant. Hence, $V_{0}$ is the dual $\mathfrak{g}$-module of itself. Suppose that $i \geq 1$. Take an element $u_{i} \in V_{i}$ such that $B_{i}\left(u_{i}, q_{-i+1}\left(\phi_{-1} \otimes \psi_{-i+1}\right)\right)=0$ for any $\phi_{-1} \in V_{-1}$ and $\psi_{-i+1} \in V_{-i+1}$. Then we have

$$
B_{i-1}\left(u_{i}\left(\Psi^{-1}\left(\phi_{-1}\right)\right), \psi_{-i+1}\right)=0 .
$$

By the induction hypothesis, $B_{i-1}$ is non-degenerate and thus we have $u_{i}\left(\Psi^{-1}\left(\phi_{-1}\right)\right)=0$ for any $\phi_{-1} \in V_{-1}$. Thus, $u_{i}=0$. Similarly, if $\psi_{-i} \in V_{-i}$ satisfies $B_{i}\left(p_{i-1}\left(v_{1} \otimes u_{i-1}\right), \psi_{-i}\right)=0$ for any $v_{1} \in V_{1}$ and $u_{i-1} \in V_{i-1}$, then we have $\psi_{-i}=0$. This completes the proof.

\subsection{Examples}

In this section, we shall give some examples.

Example 1.14. Let $m \geq 2$ be a positive integer. Let $\mathfrak{g}=\mathfrak{g l}_{1} \oplus \mathfrak{s l}_{m}$ and $V=\mathbb{C}^{m}$, where $\mathbb{C}^{m}$ is considered as the space of column vectors. We define a representation $\rho$ of $\mathfrak{g}$ on $V$ by

$$
\rho(a, A) v:=a v+A v \quad\left(a \in \mathfrak{g l}_{1}, A \in \mathfrak{s l}_{m}, v \in V\right) .
$$

The dual space $V^{*}$ is also identified with $\mathbb{C}^{m}$ and the pairing between $V$ and $V^{*}$ is given by

$$
\begin{array}{r}
\langle\cdot, \cdot\rangle: V \times V^{*} \rightarrow \mathbb{C} \\
(v, \phi) \mapsto{ }^{\mathrm{t}} v \phi .
\end{array}
$$


The representation $\rho^{*}$ of $\mathfrak{g}$ on $V^{*}$ is given by

$$
\rho^{*}(a, A) \phi=-a \phi-{ }^{\mathrm{t}} A \phi \quad\left(a \in \mathfrak{g l}_{1}, A \in \mathfrak{s l}_{m}, \phi \in V^{*}\right),
$$

where ${ }^{\mathrm{t}} A$ is the transpose of $A$. Let $B_{0}^{1}$ be a bilinear form on $\mathfrak{g}$ given by

$$
B_{0}^{1}\left((a, A),\left(a^{\prime}, A^{\prime}\right)\right):=\frac{m}{m+1} a a^{\prime}+\operatorname{Tr} A A^{\prime} \quad\left(a, a^{\prime} \in \mathfrak{g l}_{1}, A, A^{\prime} \in \mathfrak{s l}_{m}\right) .
$$

Then $\left(\mathfrak{g}, \rho, V, B_{0}^{1}\right)$ is a standard quadruplet. The $\Phi$-map of $\left(\mathfrak{g}, \rho, V, B_{0}^{1}\right)$, denoted by $\hat{\Phi}^{1}$, is given as

$$
\hat{\Phi}^{1}(v \otimes \phi)=\left(\frac{m+1}{m}{ }^{\mathrm{t}} v \phi, v^{\mathrm{t}} \phi-\frac{1}{m}{ }^{\mathrm{t}} v \phi I_{m}\right),
$$

where $I_{m}$ is the unit matrix of size $m$.

In this situation, the $n$-graduation of $\left(\mathfrak{g}, \rho, V, B_{0}^{1}\right)$ is $\{0\}$ for all $n \geq 2$. In fact, we can identify $V_{1}$ with $V$. Then, for any $v_{1}, u_{1} \in V_{1}$ and $\phi \in V^{*}$, we have

$$
\begin{aligned}
p_{1} & \left(v_{1} \otimes u_{1}\right)(\phi) \\
= & \rho_{1}\left(\frac{m+1}{m}{ }^{\mathrm{t}} v_{1} \phi, v_{1}{ }^{\mathrm{t}} \phi-\frac{1}{m}{ }^{\mathrm{t}} v_{1} \phi I_{m}\right) u_{1}-\rho_{1}\left(\frac{m+1}{m}{ }^{\mathrm{t}} u_{1} \phi, u_{1}{ }^{\mathrm{t}} \phi-\frac{1}{m}{ }^{\mathrm{t}} u_{1} \phi I_{m}\right) v_{1} \\
= & \frac{m+1}{m}{ }^{\mathrm{t}} v_{1} \phi u_{1}+v_{1}{ }^{\mathrm{t}} \phi u_{1}-\frac{1}{m}{ }^{\mathrm{t}} v_{1} \phi u_{1}-\frac{m+1}{m}{ }^{\mathrm{t}} u_{1} \phi v_{1}-u_{1}{ }^{\mathrm{t}} \phi v_{1}+\frac{1}{m}{ }^{\mathrm{t}} u_{1} \phi v_{1} \\
= & \frac{m+1}{m} u_{1}{ }^{\mathrm{t}} v_{1} \phi+v_{1}{ }^{\mathrm{t}} u_{1} \phi-\frac{1}{m} u_{1}{ }^{\mathrm{t}} v_{1} \phi-\frac{m+1}{m} v_{1}{ }^{\mathrm{t}} u_{1} \phi-u_{1}{ }^{\mathrm{t}} v_{1} \phi+\frac{1}{m} v_{1}{ }^{\mathrm{t}} u_{1} \phi \\
= & 0 .
\end{aligned}
$$

Thus, our claim holds.

Example 1.15. We retain the notation $\mathfrak{g}, \rho$ and $V$ of Example 1.14 and choose another bilinear form $B_{0}^{2}$ on $\mathfrak{g}$ given as follows:

$$
B_{0}^{2}\left((a, A),\left(a^{\prime}, A^{\prime}\right)\right):=m a a^{\prime}+\operatorname{Tr} A A^{\prime} \quad\left(a, a^{\prime} \in \mathfrak{g l}_{1}, A, A^{\prime} \in \mathfrak{s l}_{m}\right) .
$$

Then the $\Phi$-map of $\left(\mathfrak{g}, \rho, V, B_{0}^{2}\right)$, denoted by $\hat{\Phi}^{2}$, is given as

$$
\hat{\Phi}^{2}(v \otimes \phi)=\left(\frac{1}{m}{ }^{\mathrm{t}} v \phi, v^{\mathrm{t}} \phi-\frac{1}{m}^{\mathrm{t}} v \phi I_{m}\right) \quad\left(v \in V, \phi \in V^{*}\right) .
$$

Identifying $V_{1}$ with $V$, we have

$$
p_{1}\left(v_{1} \otimes u_{1}\right)(\phi)=\left(v_{1}{ }^{\mathrm{t}} u_{1}-u_{1}{ }^{\mathrm{t}} v_{1}\right) \phi \quad\left(v_{1}, u_{1} \in V_{1}, \phi \in V^{*}\right)
$$

from a similar argument to the argument in Example 1.14. Therefore, we can identify $V_{2}$ with the totality of skew-symmetric matrices of size $m$ and the representation $\rho_{2}$ on $V_{2}$ is given as follows:

$$
\rho_{2}(a, A) u_{2}=2 a u_{2}+A u_{2}+u_{2}{ }^{\mathrm{t}} A \quad\left(a \in \mathfrak{g l}_{1}, A \in \mathfrak{s l}_{m}, u_{2} \in V_{2}\right)
$$


In this situation, the $n$-graduation of $\left(\mathfrak{g}, \rho, V, B_{0}^{2}\right)$ is $\{0\}$ for all $n \geq 3$. In fact, for any $v_{1}, u_{1}, w_{1} \in V_{1}$ and $\phi \in V^{*}$, we have

$$
\begin{aligned}
& p_{2}\left(w_{1} \otimes p_{1}\left(v_{1} \otimes u_{1}\right)\right)(\phi) \\
&= \rho_{2}\left(\frac{1}{m}{ }^{\mathrm{t}} w_{1} \phi, w_{1}{ }^{\mathrm{t}} \phi-\frac{1}{m}{ }^{\mathrm{t}} w_{1} \phi I_{m}\right) p_{1}\left(v_{1} \otimes u_{1}\right)+p_{1}\left(w_{1} \otimes p_{1}\left(v_{1} \otimes u_{1}\right)(\phi)\right) \\
&= p_{1}\left(\rho_{1}\left(\frac{1}{m}{ }^{\mathrm{t}} w_{1} \phi, w_{1}{ }^{\mathrm{t}} \phi-\frac{1}{m}{ }^{\mathrm{t}} w_{1} \phi I_{m}\right) v_{1} \otimes u_{1}\right) \\
&+p_{1}\left(v_{1} \otimes \rho_{1}\left(\frac{1}{m}{ }^{\mathrm{t}} w_{1} \phi, w_{1}{ }^{\mathrm{t}} \phi-\frac{1}{m}{ }^{\mathrm{t}} w_{1} \phi I_{m}\right) u_{1}\right) \\
&+p_{1}\left(w_{1} \otimes p_{1}\left(v_{1} \otimes u_{1}\right)(\phi)\right) \\
&= p_{1}\left(w_{1}{ }^{\mathrm{t}} \phi v_{1} \otimes u_{1}\right)+p_{1}\left(v_{1} \otimes w_{1}{ }^{\mathrm{t}} \phi u_{1}\right)+p_{1}\left(w_{1} \otimes\left(v_{1}{ }^{\mathrm{t}} u_{1}-u_{1}{ }^{\mathrm{t}} v_{1}\right) \phi\right) \\
&= w_{1}{ }^{\mathrm{t}} \phi v_{1}{ }^{\mathrm{t}} u_{1}-u_{1}{ }^{\mathrm{t}} v_{1} \phi^{\mathrm{t}} w_{1}+v_{1}{ }^{\mathrm{t}} u_{1} \phi^{\mathrm{t}} w_{1}-w_{1}{ }^{\mathrm{t}} \phi u_{1}{ }^{\mathrm{t}} v_{1} \\
&+w_{1}{ }^{\mathrm{t}} \phi u_{1}{ }^{\mathrm{t}} v_{1}-v_{1}{ }^{\mathrm{t}} u_{1} \phi^{\mathrm{t}} w_{1}-w_{1}{ }^{\mathrm{t}} \phi v_{1}{ }^{\mathrm{t}} u_{1}+u_{1}{ }^{\mathrm{t}} v_{1} \phi^{\mathrm{t}} w_{1} \\
&= 0 .
\end{aligned}
$$

Thus, our claim holds.

\section{A Lie algebra associated with a standard quadruplet}

In the previous section, we constructed a family of $n$-graduations from a standard quadruplet $\left(\mathfrak{g}, \rho, V, B_{0}\right)$. In this section, we consider the direct sum of $n$-graduations and denote it by $L\left(\mathfrak{g}, \rho, V, B_{0}\right)$. Then we can define a bilinear map from $L\left(\mathfrak{g}, \rho, V, B_{0}\right) \times L\left(\mathfrak{g}, \rho, V, B_{0}\right)$ to $L\left(\mathfrak{g}, \rho, V, B_{0}\right)$. We will prove that this bilinear map satisfies the axioms of a Lie algebra. First of all, let us define the following bilinear maps.

Definition 2.1. For any $n \in \mathbb{Z}$ and the $n$-graduation $V_{n}$ of a standard quadruplet $\left(\mathfrak{g}, \rho, V, B_{0}\right)$, we define the following bilinear maps

$$
\begin{aligned}
& {[\cdot, \cdot]_{n}^{0}: V_{0} \times V_{n} \rightarrow V_{n},} \\
& {[\cdot, \cdot]_{n}^{1}: V_{1} \times V_{n} \rightarrow V_{n+1},} \\
& {[\cdot, \cdot]_{n}^{-1}: V_{-1} \times V_{n} \rightarrow V_{n-1},}
\end{aligned}
$$

by

$$
\begin{gathered}
{\left[a, x_{n}\right]_{n}^{0}:=\rho_{n}(a) x_{n},} \\
{\left[v_{1}, x_{n}\right]_{n}^{1}:= \begin{cases}p_{n}\left(v_{1} \otimes x_{n}\right) & (n \geq 0), \\
-x_{n}\left(\Phi^{-1}\left(v_{1}\right)\right) & (n \leq-1),\end{cases} } \\
{\left[\phi_{-1}, x_{n}\right]_{n}^{-1}:= \begin{cases}-x_{n}\left(\Psi^{-1}\left(\phi_{-1}\right)\right) & (n \geq 1), \\
q_{n}\left(\phi_{-1} \otimes x_{n}\right) & (n \leq 0),\end{cases} }
\end{gathered}
$$

where $a \in V_{0}, v_{1} \in V_{1}, \phi_{-1} \in V_{-1}$ and $x_{n} \in V_{n}$. 
We have the following two propositions.

Proposition 2.2. For any $n \in \mathbb{Z}, a \in V_{0}, v_{1} \in V_{1}, \phi_{-1} \in V_{-1}$ and $x_{n} \in V_{n}$, we have the following equations:

$$
\begin{aligned}
{\left[p_{0}\left(v_{1} \otimes a\right), x_{n}\right]_{n}^{1} } & =\left[v_{1},\left[a, x_{n}\right]_{n}^{0}\right]_{n}^{1}-\left[a,\left[v_{1}, x_{n}\right]_{n}^{1}\right]_{n+1}^{0}, \\
{\left[q_{0}\left(\phi_{-1} \otimes a\right), x_{n}\right]_{n}^{-1} } & =\left[\phi_{-1},\left[a, x_{n}\right]_{n}^{0}\right]_{n}^{-1}-\left[a,\left[\phi_{-1}, x_{n}\right]_{n}^{-1}\right]_{n-1}^{0} .
\end{aligned}
$$

Proof. Let us show (2.4). For $n \geq 0$, we have

$$
\begin{aligned}
{\left[p_{0}\left(v_{1} \otimes a\right), x_{n}\right]_{n}^{1} } & =\left[-\rho_{1}(a) v_{1}, x_{n}\right]_{n}^{1} \\
& =p_{n}\left(-\rho_{1}(a) v_{1} \otimes x_{n}\right) \\
& =p_{n}\left(v_{1} \otimes \rho_{n}(a) x_{n}\right)-\rho_{n+1}(a) p_{n}\left(v_{1} \otimes x_{n}\right) \\
& =\left[v_{1},\left[a, x_{n}\right]_{n}^{0}\right]_{n}^{1}-\left[a,\left[v_{1}, x_{n}\right]_{n}^{1}\right]_{n+1}^{0} .
\end{aligned}
$$

For $n \leq-1$, we have

$$
\begin{aligned}
{\left[p_{0}\left(v_{1} \otimes a\right), x_{n}\right]_{n}^{1} } & =\left[-\rho_{1}(a) v_{1}, x_{n}\right]_{n}^{1} \\
& =x_{n}\left(\rho(a)\left(\Phi^{-1}\left(v_{1}\right)\right)\right) \\
& =-\left(\rho_{n}(a) x_{n}\right)\left(\Phi^{-1}\left(v_{1}\right)\right)+\rho_{n+1}(a)\left(x_{n}\left(\Phi^{-1}\left(v_{1}\right)\right)\right) \\
& =\left[v_{1},\left[a, x_{n}\right]_{n}^{0}\right]_{n}^{1}-\left[a,\left[v_{1}, x_{n}\right]_{n}^{1}\right]_{n+1}^{0} .
\end{aligned}
$$

Thus, we have (2.4). Similarly we can obtain (2.5).

Proposition 2.3. For any $n \in \mathbb{Z}, v_{1} \in V_{1}, \phi_{-1} \in V_{-1}$ and $x_{n} \in V_{n}$, we have the following equation:

$$
\left[\phi_{-1},\left[v_{1}, x_{n}\right]_{n}^{1}\right]_{n+1}^{-1}=\left[\left[\phi_{-1}, v_{1}\right]_{1}^{-1}, x_{n}\right]_{n}^{0}+\left[v_{1},\left[\phi_{-1}, x_{n}\right]_{n}^{-1}\right]_{n-1}^{1} .
$$

Proof. For $n \geq 1$, we have

$$
\begin{aligned}
{\left[\phi_{-1},\left[v_{1}, x_{n}\right]_{n}^{1}\right]_{n+1}^{-1} } & =\left[\phi_{-1}, p_{n}\left(v_{1} \otimes x_{n}\right)\right]_{n+1}^{-1} \\
& =-p_{n}\left(v_{1} \otimes x_{n}\right)\left(\Psi^{-1}\left(\phi_{-1}\right)\right) \\
& =-\rho_{n}\left(v_{1}\left(\Psi^{-1}\left(\phi_{-1}\right)\right)\right) x_{n}-p_{n-1}\left(v_{1} \otimes x_{n}\left(\Psi^{-1}\left(\phi_{-1}\right)\right)\right) \\
& =\left[\left[\phi_{-1}, v_{1}\right]_{1}^{-1}, x_{n}\right]_{n}^{0}+\left[v_{1},\left[\phi_{-1}, x_{n}\right]_{n}^{-1}\right]_{n-1}^{1} .
\end{aligned}
$$

For $n=0$, we have

$$
\begin{aligned}
{\left[\phi_{-1},\left[v_{1}, x_{0}\right]_{0}^{1}\right]_{1}^{-1} } & =-\left[\phi_{-1}, \rho_{1}\left(x_{0}\right) v_{1}\right]_{1}^{-1} \\
& =\left(\rho_{1}\left(x_{0}\right) v_{1}\right)\left(\Psi^{-1}\left(\phi_{-1}\right)\right) \\
& =\rho_{0}\left(x_{0}\right)\left(v_{1}\left(\Psi^{-1}\left(\phi_{-1}\right)\right)-v_{1}\left(\Psi^{-1}\left(\rho_{-1}\left(x_{0}\right) \phi_{-1}\right)\right)\right. \\
& =\left[\left[\phi_{-1}, v_{1}\right]_{1}^{-1}, x_{0}\right]_{0}^{0}+\left[v_{1},\left[\phi_{-1}, x_{0}\right]_{0}^{-1}\right]_{-1}^{1} .
\end{aligned}
$$


For $n \leq-1$, we have

$$
\begin{aligned}
{\left[\phi_{-1},\left[v_{1}, x_{n}\right]_{n}^{1}\right]_{n+1}^{-1} } & =-\left[\phi_{-1}, x_{n}\left(\Phi^{-1}\left(v_{1}\right)\right)\right]_{n+1}^{-1} \\
& =-q_{n+1}\left(\phi_{-1} \otimes x_{n}\left(\Phi^{-1}\left(v_{1}\right)\right)\right) \\
& =\rho_{n}\left(\phi_{-1}\left(\Phi^{-1}\left(v_{1}\right)\right)\right) x_{n}-q_{n}\left(\phi_{-1} \otimes x_{n}\right)\left(\Phi^{-1}\left(v_{1}\right)\right) \\
& =\left[\left[\phi_{-1}, v_{1}\right]_{1}^{-1}, x_{n}\right]_{n}^{0}+\left[v_{1},\left[\phi_{-1}, x_{n}\right]_{n}^{-1}\right]_{n-1}^{1} .
\end{aligned}
$$

Thus, we have (2.6).

Definition 2.4. Assume that $i \geq 0$. For any $n \in \mathbb{Z}$, we define bilinear maps

$$
\begin{aligned}
& {[\cdot, \cdot]_{n}^{i+1}: V_{i+1} \times V_{n} \rightarrow V_{i+n+1},} \\
& {[\cdot, \cdot]_{n}^{-i-1}: V_{-i-1} \times V_{n} \rightarrow V_{-i+n-1}}
\end{aligned}
$$

by

$$
\begin{aligned}
{\left[p_{i}\left(v_{1} \otimes u_{i}\right), x_{n}\right]_{n}^{i+1}:=} & {\left[v_{1},\left[u_{i}, x_{n}\right]_{n}^{i}\right]_{i+n}^{1}-\left[u_{i},\left[v_{1}, x_{n}\right]_{n}^{1}\right]_{n+1}^{i} } \\
& \left(v_{1} \in V_{1}, u_{i} \in V_{i}, x_{n} \in V_{n}\right)
\end{aligned}
$$

and

$$
\begin{aligned}
{\left[q_{-i}\left(\phi_{-1} \otimes \psi_{-i}\right), x_{n}\right]_{n}^{-i-1}:=} & {\left[\phi_{-1},\left[\psi_{-i}, x_{n}\right]_{n}^{-i}\right]_{-i+n}^{-1}-\left[\psi_{-i},\left[\phi_{-1}, x_{n}\right]_{n}^{-1}\right]_{n-1}^{-i} } \\
& \left(\phi_{-1} \in V_{-1}, \psi_{-i} \in V_{-i}, x_{n} \in V_{n}\right)
\end{aligned}
$$

inductively.

We must prove the well-definedness of Definition 2.4. First, let us start with the case where $i=0$. For this, it is sufficient to show that the bilinear maps $[\cdot, \cdot]_{n}^{1}$ and $[\cdot, \cdot]_{n}^{-1}$ given in (2.9) and (2.10) coincide with (2.2) and (2.3), respectively. It follows from Proposition 2.2. Next, let us show the following proposition to prove the well-definedness of (2.9) for $i \geq 1$.

Proposition 2.5. Let $i \geq 0$ and assume that $[\cdot, \cdot]_{n}^{i}$ is well defined for any $n \in \mathbb{Z}$. Take elements $v_{1}^{1}, \ldots, v_{1}^{l} \in V_{1}$ and $u_{i}^{1}, \ldots, u_{i}^{l} \in V_{i}$ satisfying

$$
\sum_{s=1}^{l} p_{i}\left(v_{1}^{s} \otimes u_{i}^{s}\right)=0 .
$$

Then for any $n \in \mathbb{Z}$ and $x_{n} \in V_{n}$, we have

$$
\sum_{s=1}^{l}\left(\left[v_{1}^{s},\left[u_{i}^{s}, x_{n}\right]_{n}^{i}\right]_{i+n}^{1}-\left[u_{i}^{s},\left[v_{1}^{s}, x_{n}\right]_{n}^{1}\right]_{n+1}^{i}\right)=0
$$

and hence we obtain that (2.9) is well defined.

Proof. We argue by induction on $i$. For $i=0$. By Proposition 2.2, we have our result for any $n \in \mathbb{Z}$. Moreover, by the definition and Proposition 2.3, we have the following equations:

$$
\begin{aligned}
& {\left[a,\left[u_{1}, x_{n}\right]_{n}^{1}\right]_{n+1}^{0}=\left[\left[a, u_{1}\right]_{1}^{0}, x_{n}\right]_{n}^{1}+\left[u_{1},\left[a, x_{n}\right]_{n}^{0}\right]_{n}^{1},} \\
& {\left[\phi_{-1},\left[u_{1}, x_{n}\right]_{n}^{1}\right]_{n+1}^{-1}=\left[\left[\phi_{-1}, u_{1}\right]_{1}^{-1}, x_{n}\right]_{n}^{0}+\left[u_{1},\left[\phi_{-1}, x_{n}\right]_{n}^{-1}\right]_{n-1}^{1},} \\
& {\left[u_{1}, a\right]_{0}^{1}=-\left[a, u_{1}\right]_{1}^{0}=-\rho_{1}(a) u_{1},}
\end{aligned}
$$




$$
\begin{aligned}
& {\left[u_{1}, v_{1}\right]_{1}^{1}=-\left[v_{1}, u_{1}\right]_{1}^{1}=-p_{1}\left(v_{1} \otimes u_{1}\right),} \\
& {\left[u_{1}, \phi_{-1}\right]_{-1}^{1}=-\left[\phi_{-1}, u_{1}\right]_{1}^{-1}=u_{1}\left(\Psi^{-1}\left(\phi_{-1}\right)\right),}
\end{aligned}
$$

where $a \in V_{0}, v_{1}, u_{1} \in V_{1}, \phi_{-1} \in V_{-1}$ and $x_{n} \in V_{n}$.

For $i \geq 1$. We assume that the bilinear map $[\cdot, \cdot]_{n}^{i}$ is well defined for any $n \in \mathbb{Z}$ and satisfies the following equations:

$$
\begin{aligned}
& {\left[a,\left[u_{i}, x_{n}\right]_{n}^{i}\right]_{i+n}^{0}=\left[\left[a, u_{i}\right]_{i}^{0}, x_{n}\right]_{n}^{i}+\left[u_{i},\left[a, x_{n}\right]_{n}^{0}\right]_{n}^{i},} \\
& {\left[\phi_{-1},\left[u_{i}, x_{n}\right]_{n}^{i}\right]_{i+n}^{-1}=\left[\left[\phi_{-1}, u_{i}\right]_{i}^{-1}, x_{n}\right]_{n}^{i-1}+\left[u_{i},\left[\phi_{-1}, x_{n}\right]_{n}^{-1}\right]_{n-1}^{i},} \\
& {\left[u_{i}, a\right]_{0}^{i}=-\left[a, u_{i}\right]_{i}^{0}=-\rho_{i}(a) u_{i},} \\
& {\left[u_{i}, v_{1}\right]_{1}^{i}=-\left[v_{1}, u_{i}\right]_{i}^{1}=-p_{i}\left(v_{1} \otimes u_{i}\right),} \\
& {\left[u_{i}, \phi_{-1}\right]_{-1}^{i}=-\left[\phi_{-1}, u_{i}\right]_{i}^{-1}=u_{i}\left(\Psi^{-1}\left(\phi_{-1}\right)\right),}
\end{aligned}
$$

where $a \in V_{0}, v_{1} \in V_{1}, u_{i} \in V_{i}, \phi_{-1} \in V_{-1}$ and $x_{n} \in V_{n}$.

We fix $i$ and argue by induction on $n$.

(1) The case where $n \geq 0$.

For $n=0$, by the induction hypothesis on $i$, we have

$$
\begin{aligned}
& {\left[v_{1},\left[u_{i}, x_{0}\right]_{0}^{i}\right]_{i}^{1}-\left[u_{i},\left[v_{1}, x_{0}\right]_{0}^{1}\right]_{1}^{i}} \\
& \quad=-\left[v_{1}, \rho_{i}\left(x_{0}\right) u_{i}\right]_{i}^{1}+\left[u_{i}, \rho_{1}\left(x_{0}\right) v_{1}\right]_{1}^{i} \\
& \quad=-p_{i}\left(v_{1} \otimes \rho_{i}\left(x_{0}\right) u_{i}\right)-p_{i}\left(\rho_{1}\left(x_{0}\right) v_{1} \otimes u_{i}\right) \\
& \quad=-\rho_{i+1}\left(x_{0}\right) p_{i}\left(v_{1} \otimes u_{i}\right)
\end{aligned}
$$

where $v_{1} \in V_{1}, u_{i} \in V_{i}$ and $x_{0} \in V_{0}$. Thus, we have our result immediately.

For $n \geq 1$. Note that by (2.19), we have

$$
\left[u_{i}, y_{m}\right]_{m}^{i}(\phi)=\left[u_{i}(\phi), y_{m}\right]_{m}^{i-1}+\left[u_{i}, y_{m}(\phi)\right]_{m-1}^{i}
$$

for any $m \geq 1, u_{i} \in V_{i}, y_{m} \in V_{m}$ and $\phi \in V^{*}$. Hence, we have

$$
\begin{aligned}
{\left[v_{1},\right.} & {\left.\left[u_{i}, x_{n}\right]_{n}^{i}\right]_{i+n}^{1}(\phi)-\left[u_{i},\left[v_{1}, x_{n}\right]_{n}^{1}\right]_{n+1}^{i}(\phi) } \\
= & p_{i+n}\left(v_{1} \otimes\left[u_{i}, x_{n}\right]_{n}^{i}\right)(\phi)-\left[u_{i}, p_{n}\left(v_{1} \otimes x_{n}\right)\right]_{n+1}^{i}(\phi) \\
= & \rho_{i+n}\left(v_{1}(\phi)\right)\left[u_{i}, x_{n}\right]_{n}^{i} \\
& +p_{i+n-1}\left(v_{1} \otimes\left(\left[u_{i}(\phi), x_{n}\right]_{n}^{i-1}+\left[u_{i}, x_{n}(\phi)\right]_{n-1}^{i}\right)\right) \\
& -\left[u_{i}(\phi), p_{n}\left(v_{1} \otimes x_{n}\right)\right]_{n+1}^{i-1} \\
& -\left[u_{i}, \rho_{n}\left(v_{1}(\phi)\right) x_{n}+p_{n-1}\left(v_{1} \otimes x_{n}(\phi)\right)\right]_{n}^{i} \\
= & {\left[\rho_{i}\left(v_{1}(\phi)\right) u_{i}, x_{n}\right]_{n}^{i} } \\
& +p_{i+n-1}\left(v_{1} \otimes\left[u_{i}(\phi), x_{n}\right]_{n}^{i-1}\right)-\left[u_{i}(\phi), p_{n}\left(v_{1} \otimes x_{n}\right)\right]_{n+1}^{i-1} \\
& +p_{i+n-1}\left(v_{1} \otimes\left[u_{i}, x_{n}(\phi)\right]_{n-1}^{i}\right)-\left[u_{i}, p_{n-1}\left(v_{1} \otimes x_{n}(\phi)\right)\right]_{n}^{i}
\end{aligned}
$$




$$
\begin{aligned}
= & {\left[\rho_{i}\left(v_{1}(\phi)\right) u_{i}, x_{n}\right]_{n}^{i}+\left[p_{i-1}\left(v_{1} \otimes u_{i}(\phi)\right), x_{n}\right]_{n}^{i} } \\
& +p_{i+n-1}\left(v_{1} \otimes\left[u_{i}, x_{n}(\phi)\right]_{n-1}^{i}\right)-\left[u_{i}, p_{n-1}\left(v_{1} \otimes x_{n}(\phi)\right)\right]_{n}^{i} \\
= & {\left[p_{i}\left(v_{1} \otimes u_{i}\right)(\phi), x_{n}\right]_{n}^{i} } \\
& +\left[v_{1},\left[u_{i}, x_{n}(\phi)\right]_{n-1}^{i}\right]_{i+n-1}^{1}-\left[u_{i},\left[v_{1}, x_{n}(\phi)\right]_{n-1}^{1}\right]_{n}^{i},
\end{aligned}
$$

where $v_{1} \in V_{1}, u_{i} \in V_{i}, x_{n} \in V_{n}$ and $\phi \in V^{*}$. Here, by the induction hypotheses on $i$ and $n$, if $v_{1}^{1}, \ldots, v_{1}^{l} \in V_{1}$ and $u_{i}^{1}, \ldots, u_{i}^{l} \in V_{i}$ satisfy

$$
\sum_{s=1}^{l} p_{i}\left(v_{1}^{s} \otimes u_{i}^{s}\right)=0
$$

then we have

$$
\sum_{s=1}^{l}\left[p_{i}\left(v_{1}^{s} \otimes u_{i}^{s}\right)(\phi), x_{n}\right]_{n}^{i}=0
$$

and

$$
\sum_{s=1}^{l}\left(\left[v_{1}^{s},\left[u_{i}^{s}, x_{n}(\phi)\right]_{n-1}^{i}\right]_{n+i-1}^{1}-\left[u_{i}^{s},\left[v_{1}^{s} \otimes x_{n}(\phi)\right]_{n-1}^{1}\right]_{n}^{i}\right)=0,
$$

respectively. Therefore, we have our result for any $n \geq 0$.

(2) The case where $n \leq-1$.

For $n=-1$, by the induction hypothesis on $i$, we have

$$
\begin{aligned}
{\left[v_{1},\right.} & {\left.\left[u_{i}, x_{-1}\right]_{-1}^{i}\right]_{i-1}^{1}-\left[u_{i},\left[v_{1}, x_{-1}\right]_{-1}^{1}\right]_{0}^{i} } \\
& =\left[v_{1}, u_{i}\left(\Psi^{-1}\left(x_{-1}\right)\right)\right]_{i-1}^{1}-\left[u_{i}, v_{1}\left(\Psi^{-1}\left(x_{-1}\right)\right)\right]_{0}^{i} \\
& =p_{i-1}\left(v_{1} \otimes u_{i}\left(\Psi^{-1}\left(x_{-1}\right)\right)\right)+\rho_{i}\left(v_{1}\left(\Psi^{-1}\left(x_{-1}\right)\right)\right) u_{i} \\
& =p_{i}\left(v_{1} \otimes u_{i}\right)\left(\Psi^{-1}\left(x_{-1}\right)\right),
\end{aligned}
$$

where $v_{1} \in V_{1}, u_{i} \in V_{i}$ and $x_{-1} \in V_{-1}$. Thus, we have our result immediately.

For $n \leq-2$. Note that by (2.19), we have

$$
\left[u_{i}, q_{m+1}\left(\phi_{-1} \otimes y_{m+1}\right)\right]_{m}^{i}=\left[u_{i}\left(\Psi^{-1}\left(\phi_{-1}\right)\right), y_{m+1}\right]_{m+1}^{i-1}+\left[\phi_{-1},\left[u_{i}, y_{m+1}\right]_{m+1}^{i}\right]_{i+m+1}^{-1}
$$

for any $m \leq-1, u_{i} \in V_{i}, \phi_{-1} \in V_{-1}$ and $y_{m+1} \in V_{m+1}$. Hence, by the induction hypotheses on $i$ and $n$ and Proposition 2.3, we have

$$
\begin{aligned}
{\left[v_{1},\right.} & {\left.\left[u_{i}, q_{n+1}\left(\phi_{-1} \otimes x_{n+1}\right)\right]_{n}^{i}\right]_{i+n}^{1}-\left[u_{i},\left[v_{1}, q_{n+1}\left(\phi_{-1} \otimes x_{n+1}\right)\right]_{n}^{1}\right]_{n+1}^{i} } \\
= & {\left[v_{1},\left[u_{i}\left(\Psi^{-1}\left(\phi_{-1}\right)\right), x_{n+1}\right]_{n+1}^{i-1}\right]_{i+n}^{1}+\left[v_{1},\left[\phi_{-1},\left[u_{i}, x_{n+1}\right]_{n+1}^{i}\right]_{i+n+1}^{-1}\right]_{i+n}^{1} } \\
& +\left[u_{i}, q_{n+1}\left(\phi_{-1} \otimes x_{n+1}\right)\left(\Phi^{-1}\left(v_{1}\right)\right)\right]_{n+1}^{i} \\
= & {\left[p_{i-1}\left(v_{1} \otimes u_{i}\left(\Psi^{-1}\left(\phi_{-1}\right)\right)\right), x_{n+1}\right]_{n+1}^{i}-\left[u_{i}\left(\Psi^{-1}\left(\phi_{-1}\right)\right), x_{n+1}\left(\Phi^{-1}\left(v_{1}\right)\right)\right]_{n+2}^{i-1} } \\
& +\left[v_{1}\left(\Psi^{-1}\left(\phi_{-1}\right)\right),\left[u_{i}, x_{n+1}\right]_{n+1}^{i}\right]_{i+n+1}^{0}+\left[\phi_{-1},\left[v_{1},\left[u_{i}, x_{n+1}\right]_{n+1}^{i}\right]_{i+n+1}^{1}\right]_{i+n+2}^{-1} \\
& +\left[u_{i}, \rho_{n+1}\left(\phi_{-1}\left(\Phi^{-1}\left(v_{1}\right)\right)\right) x_{n+1}\right]_{n+1}^{i}+\left[u_{i}, q_{n+2}\left(\phi_{-1} \otimes x_{n+1}\left(\Phi^{-1}\left(v_{1}\right)\right)\right)\right]_{n+1}^{i}
\end{aligned}
$$




$$
\begin{aligned}
= & {\left[p_{i-1}\left(v_{1} \otimes u_{i}\left(\Psi^{-1}\left(\phi_{-1}\right)\right)\right), x_{n+1}\right]_{n+1}^{i}-\left[u_{i}\left(\Psi^{-1}\left(\phi_{-1}\right)\right), x_{n+1}\left(\Phi^{-1}\left(v_{1}\right)\right)\right]_{n+2}^{i-1} } \\
& +\left[\rho_{i}\left(v_{1}\left(\Psi^{-1}\left(\phi_{-1}\right)\right)\right) u_{i}, x_{n+1}\right]_{n+1}^{i}+\left[u_{i}, \rho_{n+1}\left(v_{1}\left(\Psi^{-1}\left(\phi_{-1}\right)\right)\right) x_{n+1}\right]_{n+1}^{i} \\
& +\left[\phi_{-1},\left[v_{1},\left[u_{i}, x_{n+1}\right]_{n+1}^{i}\right]_{i+n+1}^{1}\right]_{i+n+2}^{-1}+\left[u_{i}, \rho_{n+1}\left(\phi_{-1}\left(\Phi^{-1}\left(v_{1}\right)\right)\right) x_{n+1}\right]_{n+1}^{i} \\
& +\left[u_{i}\left(\Psi^{-1}\left(\phi_{-1}\right)\right), x_{n+1}\left(\Phi^{-1}\left(v_{1}\right)\right)\right]_{n+2}^{i-1}+\left[\phi_{-1},\left[u_{i}, x_{n+1}\left(\Phi^{-1}\left(v_{1}\right)\right)\right]_{n+2}^{i}\right]_{i+n+2}^{-1} \\
= & {\left[p_{i-1}\left(v_{1} \otimes u_{i}\left(\Psi^{-1}\left(\phi_{-1}\right)\right)\right), x_{n+1}\right]_{n+1}^{i}+\left[\rho_{i}\left(v_{1}\left(\Psi^{-1}\left(\phi_{-1}\right)\right)\right) u_{i}, x_{n+1}\right]_{n+1}^{i} } \\
& +\left[\phi_{-1},\left[v_{1},\left[u_{i}, x_{n+1}\right]_{n+1}^{i}\right]_{i+n+1}^{1}\right]_{i+n+2}^{-1}-\left[\phi_{-1},\left[u_{i},\left[v_{1}, x_{n+1}\right]_{n+1}^{1}\right]_{n+2}^{i}\right]_{i+n+2}^{-1} \\
= & {\left[p_{i}\left(v_{1} \otimes u_{i}\right)\left(\Psi^{-1}\left(\phi_{-1}\right)\right), x_{n+1}\right]_{n+1}^{i} } \\
& +\left[\phi_{-1},\left(\left[v_{1},\left[u_{i}, x_{n+1}\right]_{n+1}^{i}\right]_{i+n+1}^{1}-\left[u_{i},\left[v_{1}, x_{n+1}\right]_{n+1}^{1}\right]_{n+2}^{i}\right)\right]_{i+n+2}^{-1},
\end{aligned}
$$

where $v_{1} \in V_{1}, u_{i} \in V_{i}, x_{n+1} \in V_{n+1}$ and $\phi_{-1} \in V_{-1}$. Here, by the induction hypotheses on $i$ and $n$, if $v_{1}^{1}, \ldots, v_{1}^{l} \in V_{1}$ and $u_{i}^{1}, \ldots, u_{i}^{l} \in V_{i}$ satisfy

$$
\sum_{s=1}^{l} p_{i}\left(v_{1}^{s} \otimes u_{i}^{s}\right)=0
$$

then we have

$$
\sum_{s=1}^{l}\left[p_{i}\left(v_{1}^{s} \otimes u_{i}^{s}\right)\left(\Psi^{-1}\left(\phi_{-1}\right)\right), x_{n+1}\right]_{n+1}^{i}=0
$$

and

$$
\sum_{s=1}^{l}\left[\phi_{-1},\left(\left[v_{1}^{s},\left[u_{i}^{s}, x_{n+1}\right]_{n+1}^{i}\right]_{i+n+1}^{1}-\left[u_{i}^{s},\left[v_{1}^{s}, x_{n+1}\right]_{n+1}^{1}\right]_{n+2}^{i}\right)\right]_{i+n+2}^{-1}=0,
$$

respectively. Therefore, we have our result for any $n \leq-1$ and we obtain a well-defined bilinear map $[\cdot, \cdot]_{n}^{i+1}$ for all $n \in \mathbb{Z}$.

In order to complete the proof, let us show that the bilinear maps $[\cdot, \cdot]_{n}^{i+1}(n \in \mathbb{Z})$ satisfy the following equations:

$$
\begin{aligned}
& {\left[a,\left[u_{i+1}, x_{n}\right]_{n}^{i+1}\right]_{i+n+1}^{0}=\left[\left[a, u_{i+1}\right]_{i+1}^{0}, x_{n}\right]_{n}^{i+1}+\left[u_{i+1},\left[a, x_{n}\right]_{n}^{0}\right]_{n}^{i+1},} \\
& {\left[\phi_{-1},\left[u_{i+1}, x_{n}\right]_{n}^{i+1}\right]_{i+n+1}^{-1}=\left[\left[\phi_{-1}, u_{i+1}\right]_{i+1}^{-1}, x_{n}\right]_{n}^{i}+\left[u_{i+1},\left[\phi_{-1}, x_{n}\right]_{n}^{-1}\right]_{n-1}^{i+1},} \\
& {\left[u_{i+1}, a\right]_{0}^{i+1}=-\left[a, u_{i+1}\right]_{i+1}^{0}=-\rho_{i+1}(a) u_{i+1},} \\
& {\left[u_{i+1}, v_{1}\right]_{1}^{i+1}=-\left[v_{1}, u_{i+1}\right]_{i+1}^{1}=-p_{i+1}\left(v_{1} \otimes u_{i+1}\right),} \\
& {\left[u_{i+1}, \phi_{-1}\right]_{-1}^{i+1}=-\left[\phi_{-1}, u_{i+1}\right]_{i+1}^{-1}=u_{i+1}\left(\Psi^{-1}\left(\phi_{-1}\right)\right),}
\end{aligned}
$$

where $a \in V_{0}, v_{1} \in V_{1}, u_{i+1} \in V_{i+1}, \phi_{-1} \in V_{-1}$ and $x_{n} \in V_{n}$.

Equations (2.31) and (2.33) follow from (2.23) and (2.26), respectively.

Let us show (2.29). We can assume that $u_{i+1}=p_{i}\left(v_{1} \otimes u_{i}\right)$ for some $v_{1} \in V_{1}$ and $u_{i} \in V_{i}$ without loss of generality. Then we have

$$
\begin{aligned}
{[a,} & {\left.\left[p_{i}\left(v_{1} \otimes u_{i}\right), x_{n}\right]_{n}^{i+1}\right]_{i+n+1}^{0} } \\
= & {\left[a,\left[v_{1},\left[u_{i}, x_{n}\right]_{n}^{i}\right]_{i+n}^{1}\right]_{i+n+1}^{0}-\left[a,\left[u_{i},\left[v_{1}, x_{n}\right]_{n}^{1}\right]_{n+1}^{i}\right]_{i+n+1}^{0} } \\
= & {\left[\rho_{1}(a) v_{1},\left[u_{i}, x_{n}\right]_{n}^{i}\right]_{i+n}^{1}+\left[v_{1},\left[\rho_{i}(a) u_{i}, x_{n}\right]_{n}^{i}\right]_{i+n}^{1}+\left[v_{1},\left[u_{i}, \rho_{n}(a) x_{n}\right]_{n}^{i}\right]_{i+n}^{1} } \\
& -\left[\rho_{i}(a) u_{i},\left[v_{1}, x_{n}\right]_{n}^{1}\right]_{n+1}^{i}-\left[u_{i},\left[\rho_{1}(a) v_{1}, x_{n}\right]_{n}^{1}\right]_{n+1}^{i}-\left[u_{i},\left[v_{1}, \rho_{n}(a) x_{n}\right]_{n}^{1}\right]_{n+1}^{i}
\end{aligned}
$$




$$
\begin{aligned}
= & {\left[\rho_{1}(a) v_{1},\left[u_{i}, x_{n}\right]_{n}^{i}\right]_{i+n}^{1}-\left[u_{i},\left[\rho_{1}(a) v_{1}, x_{n}\right]_{n}^{1}\right]_{n+1}^{i} } \\
& +\left[v_{1},\left[\rho_{i}(a) u_{i}, x_{n}\right]_{n}^{i}\right]_{i+n}^{1}-\left[\rho_{i}(a) u_{i},\left[v_{1}, x_{n}\right]_{n}^{1}\right]_{n+1}^{i} \\
& +\left[v_{1},\left[u_{i}, \rho_{n}(a) x_{n}\right]_{n}^{i}\right]_{i+n}^{1}-\left[u_{i},\left[v_{1}, \rho_{n}(a) x_{n}\right]_{n}^{1}\right]_{n+1}^{i} \\
= & {\left[p_{i}\left(\rho_{1}(a) v_{1} \otimes u_{i}\right), x_{n}\right]_{n}^{i+1}+\left[p_{i}\left(v_{1} \otimes \rho_{i}(a) u_{i}\right), x_{n}\right]_{n}^{i+1} } \\
& +\left[p_{i}\left(v_{1} \otimes u_{i}\right), \rho_{n}(a) x_{n}\right]_{n}^{i+1} \\
= & {\left[\left[a, p_{i}\left(v_{1} \otimes u_{i}\right)\right]_{i+1}^{0}, x_{n}\right]_{n}^{i+1}+\left[p_{i}\left(v_{1} \otimes u_{i}\right),\left[a, x_{n}\right]_{n}^{0}\right]_{n}^{i+1} . }
\end{aligned}
$$

Thus, (2.29) holds.

Let us show (2.30). If $n \neq 0,(2.30)$ follows from (2.25) and (2.28). The case where $n=0$ is obtained from (2.31) and (2.33). Thus (2.30) holds.

Finally, let us show (2.32). By (2.30) and (2.31), for any $\phi \in V^{*}$, we have

$$
\begin{aligned}
{\left[u_{i+1}, v_{1}\right]_{1}^{i+1}(\phi) } & =\left[u_{i+1}(\phi), v_{1}\right]_{1}^{i}+\left[u_{i+1}, v_{1}(\phi)\right]_{0}^{i+1} \\
& =-p_{i}\left(v_{1} \otimes u_{i+1}(\phi)\right)-\rho_{i+1}\left(v_{1}(\phi)\right) u_{i+1} \\
& =-p_{i+1}\left(v_{1} \otimes u_{i+1}\right)(\phi) .
\end{aligned}
$$

Thus, (2.32) holds. This completes the proof.

In the same way, we can prove the following proposition.

Proposition 2.6. Assume that $j \geq 0$ and $[\cdot, \cdot]_{n}^{-j}$ is well defined for any $n \in \mathbb{Z}$. Take elements $\phi_{-1}^{1}, \ldots, \phi_{-1}^{l} \in V_{-1}$ and $\psi_{-j}^{1}, \ldots, \psi_{-j}^{l} \in V_{-j}$ satisfying

$$
\sum_{s=1}^{l} q_{-j}\left(\phi_{-1}^{s} \otimes \psi_{-j}^{s}\right)=0 .
$$

Then for any $x_{n} \in V_{n}$, we have

$$
\sum_{s=1}^{l}\left(\left[\phi_{-1}^{s},\left[\psi_{-j}^{s}, x_{n}\right]_{n}^{-j}\right]_{-j+n}^{-1}-\left[\psi_{-j}^{s},\left[\phi_{-1}^{s}, x_{n}\right]_{n}^{-1}\right]_{n-1}^{-j}\right)=0
$$

and, hence, we obtain a well-defined bilinear map

$$
[\cdot, \cdot]_{n}^{-j-1}: V_{-j-1} \times V_{n} \rightarrow V_{-j+n-1}
$$

satisfying the following equations:

$$
\begin{aligned}
& {\left[a,\left[\psi_{-j-1}, x_{n}\right]_{n}^{-j-1}\right]_{-j+n-1}^{0}=\left[\left[a, \psi_{-j-1}\right]_{-j-1}^{0}, x_{n}\right]_{n}^{-j-1}+\left[\psi_{-j-1},\left[a, x_{n}\right]_{n}^{0}\right]_{n}^{-j-1}} \\
& {\left[v_{1},\left[\psi_{-j-1}, x_{n}\right]_{n}^{-j-1}\right]_{-j+n-1}^{1}=\left[\left[v_{1}, \psi_{-j-1}\right]_{-j-1}^{1}, x_{n}\right]_{n}^{-j}+\left[\psi_{-j-1},\left[v_{1}, x_{n}\right]_{n}^{1}\right]_{n+1}^{-j-1}} \\
& {\left[\psi_{-j-1}, a\right]_{0}^{-j-1}=-\left[a, \psi_{-j-1}\right]_{-j-1}^{0}=-\rho_{-j-1}(a) \psi_{-j-1}} \\
& {\left[\psi_{-j-1}, \phi_{-1}\right]_{-1}^{-j-1}=-\left[\phi_{-1}, \psi_{-j-1}\right]_{-j-1}^{-1}=-q_{-j-1}\left(\phi_{-1} \otimes \psi_{-j-1}\right)} \\
& {\left[\psi_{-j-1}, v_{1}\right]_{1}^{-j-1}=-\left[v_{1}, \psi_{-j-1}\right]_{-j-1}^{1}=\psi_{-j-1}\left(\Phi^{-1}\left(v_{1}\right)\right)}
\end{aligned}
$$

where $a \in V_{0}, v_{1} \in V_{1}, \phi_{-1} \in V_{-1}, \psi_{-j-1} \in V_{-j-1}$ and $x_{n} \in V_{n}$. 
Then Propositions 2.5 and 2.6 are summarized as follows.

Definition 2.7. We put

$$
L\left(\mathfrak{g}, \rho, V, B_{0}\right):=\bigoplus_{n \in \mathbb{Z}} V_{n}
$$

and define a bilinear map

$$
[\cdot, \cdot]: L\left(\mathfrak{g}, \rho, V, B_{0}\right) \times L\left(\mathfrak{g}, \rho, V, B_{0}\right) \rightarrow L\left(\mathfrak{g}, \rho, V, B_{0}\right)
$$

by

$$
\left[x_{n}, y_{m}\right]:=\left[x_{n}, y_{m}\right]_{m}^{n}
$$

where $x_{n} \in V_{n}$ and $y_{m} \in V_{m}$.

PROPOSITION 2.8. The bilinear map $[\cdot, \cdot]$ satisfies the following equations:

$$
\begin{aligned}
& {[a, x]=-[x, a],} \\
& {\left[v_{1}, x\right]=-\left[x, v_{1}\right],} \\
& {\left[\phi_{-1}, x\right]=-\left[x, \phi_{-1}\right],} \\
& {[a,[x, y]]=[[a, x], y]+[x,[a, y]],} \\
& {\left[y,\left[v_{1}, x\right]\right]=\left[v_{1},[y, x]\right]-\left[\left[v_{1}, y\right], x\right],} \\
& {\left[y,\left[\phi_{-1}, x\right]\right]=\left[\phi_{-1},[y, x]\right]-\left[\left[\phi_{-1}, y\right], x\right],}
\end{aligned}
$$

where $a \in V_{0}, v_{1} \in V_{1}, \phi_{-1} \in V_{-1}$ and $x, y \in L\left(\mathfrak{g}, \rho, V, B_{0}\right)$.

Let us show that $[\cdot, \cdot]$ satisfies the axioms of a Lie algebra.

Proposition 2.9. For any $x, y \in L\left(\mathfrak{g}, \rho, V, B_{0}\right)$, we have

$$
[x, y]+[y, x]=0 .
$$

Namely, $[\cdot, \cdot]$ is skew-symmetric.

Proof. Without loss of generality, it is sufficient to show this in the cases where $x=x_{n} \in V_{n}$ for some $n \in \mathbb{Z}$. We assume that $n \geq 0$ and argue by induction on $n$.

For $n=0$, our claim follows from Proposition 2.8.

For $n \geq 1$, without loss of generality, we can assume that there exists $v_{1} \in V_{1}$ and $x_{n-1} \in V_{n-1}$ such that $x_{n}=p_{n-1}\left(v_{1} \otimes x_{n-1}\right)$. Then, by (2.9) and (2.47), we have

$$
\begin{aligned}
& {\left[p_{n-1}\left(v_{1} \otimes x_{n-1}\right), y\right]+\left[y, p_{n-1}\left(v_{1} \otimes x_{n-1}\right)\right]} \\
& \quad=\left[v_{1},\left[x_{n-1}, y\right]\right]-\left[x_{n-1},\left[v_{1}, y\right]\right]+\left[v_{1},\left[y, x_{n-1}\right]\right]-\left[\left[v_{1}, y\right], x_{n-1}\right] .
\end{aligned}
$$

By the induction hypothesis, we have

$$
\left[x_{n-1}, y\right]+\left[y, x_{n-1}\right]=0
$$

and

$$
\left[x_{n-1},\left[v_{1}, y\right]\right]+\left[\left[v_{1}, y\right], x_{n-1}\right]=0 .
$$

Hence, our claim holds. Similarly we can obtain the proof of the case where $n \leq-1$. This completes the proof. 
Proposition 2.10. For any $x, y, z \in L\left(\mathfrak{g}, \rho, V, B_{0}\right)$, we have

$$
[x,[y, z]]=[[x, y], z]+[y,[x, z]] .
$$

Namely, $[\cdot, \cdot]$ satisfies the Jacobi identity.

Proof. Without loss of generality, it is sufficient to show in the case where $x=x_{n} \in V_{n}$ for some $n \in \mathbb{Z}$. We assume $n \geq 0$ and argue by induction on $n$.

For $n=0$ and 1, our claim follows from Proposition 2.8.

For $n \geq 2$, without loss of generality, we can assume that $x_{n}=p_{n-1}\left(v_{1} \otimes x_{n-1}\right)$ for some $v_{1} \in V_{1}$ and $x_{n-1} \in V_{n-1}$. Then, by the induction hypothesis, we have

$$
\begin{aligned}
{\left[p_{n-1}\right.} & \left.\left(v_{1} \otimes x_{n-1}\right),[y, z]\right] \\
= & {\left[v_{1},\left[x_{n-1},[y, z]\right]\right]-\left[x_{n-1},\left[v_{1},[y, z]\right]\right] } \\
= & {\left[v_{1},\left[\left[x_{n-1}, y\right], z\right]\right]+\left[v_{1},\left[y,\left[x_{n-1}, z\right]\right]\right] } \\
& -\left[x_{n-1},\left[\left[v_{1}, y\right], z\right]\right]-\left[x_{n-1},\left[y,\left[v_{1}, z\right]\right]\right] \\
= & {\left[v_{1},\left[\left[x_{n-1}, y\right], z\right]\right]+\left[y,\left[v_{1},\left[x_{n-1}, z\right]\right]\right]+\left[\left[v_{1}, y\right],\left[x_{n-1}, z\right]\right] } \\
& -\left[x_{n-1},\left[\left[v_{1}, y\right], z\right]\right]-\left[y,\left[x_{n-1},\left[v_{1}, z\right]\right]\right]-\left[\left[x_{n-1}, y\right],\left[v_{1}, z\right]\right] \\
= & {\left[\left[v_{1},\left[x_{n-1}, y\right]\right], z\right]-\left[\left[x_{n-1},\left[v_{1}, y\right]\right], z\right]+\left[y,\left[p_{n-1}\left(v_{1} \otimes x_{n-1}\right), z\right]\right] } \\
= & {\left[\left[p_{n-1}\left(v_{1} \otimes x_{n-1}\right), y\right], z\right]+\left[y,\left[p_{n-1}\left(v_{1} \otimes x_{n-1}\right), z\right]\right] . }
\end{aligned}
$$

Thus, we have our claim. We can also obtain the proof of the case where $n \leq-1$ by the same argument. This completes the proof.

By Propositions 2.9 and 2.10, we have the following theorem.

THEOREM 2.11. The vector space $L\left(\mathfrak{g}, \rho, V, B_{0}\right)$ is a $\mathbb{Z}$-graded Lie algebra with Lie bracket $[\cdot, \cdot]$. We call the Lie algebra thus obtained the Lie algebra associated with $\left(\mathfrak{g}, \rho, V, B_{0}\right)$.

In particular, for a given reductive Lie algebra $\mathfrak{g}$ and its finite-dimensional completely reducible representation $\rho$ on $V$, there exists a graded Lie algebra $\sum_{n \in \mathbb{Z}} \mathfrak{g}_{n}$ such that the Lie subalgebra $\mathfrak{g}_{0}$ coincides with $\mathfrak{g}$ and the adjoint representation of $\mathfrak{g}_{0}$ on $\mathfrak{g}_{1}$ is isomorphic to $(\rho, V)$.

In the next section, we construct a bilinear form on $L\left(\mathfrak{g}, \rho, V, B_{0}\right)=\bigoplus_{n \in \mathbb{Z}} V_{n}$ such that the restriction to $V_{0} \times V_{0}$ coincides with $B_{0}$.

\section{Some properties of $L\left(\mathfrak{g}, \rho, V, B_{0}\right)$}

\subsection{A bilinear form on $L\left(\mathfrak{g}, \rho, V, B_{0}\right)$}

In this section, we consider the structure of the Lie algebra $L\left(\mathfrak{g}, \rho, V, B_{0}\right)$ associated with a standard quadruplet $\left(\mathfrak{g}, \rho, V, B_{0}\right)$. First of all, we shall construct a non-degenerate symmetric $L\left(\mathfrak{g}, \rho, V, B_{0}\right)$-invariant bilinear form on $L\left(\mathfrak{g}, \rho, V, B_{0}\right)$. 
Definition 3.1. We define a bilinear form $B$ on $L\left(\mathfrak{g}, \rho, V, B_{0}\right)$ by

$$
B\left(\sum_{n \in \mathbb{Z}} x_{n}, \sum_{m \in \mathbb{Z}} y_{m}\right):=\sum_{s \geq 0} B_{s}\left(x_{s}, y_{-s}\right)+\sum_{t \leq-1} B_{-t}\left(y_{-t}, x_{t}\right)
$$

where $x_{n} \in V_{n}$ and $y_{m} \in V_{m}$.

PROPOSITION 3.2. The bilinear form $B$ is non-degenerate symmetric and $L\left(\mathfrak{g}, \rho, V, B_{0}\right)$ invariant.

Proof. It can be easily proved that $B$ is non-degenerate and symmetric. Let us prove that $B$ is $L\left(\mathfrak{g}, \rho, V, B_{0}\right)$-invariant. For this, let us show that we have

$$
\begin{gathered}
B\left(\left[u_{i}, x_{n}\right], y_{m}\right)=B\left(x_{n},\left[y_{m}, u_{i}\right]\right), \\
B\left(\left[\psi_{-i}, x_{n}\right], y_{m}\right)=B\left(x_{n},\left[y_{m}, \psi_{-i}\right]\right),
\end{gathered}
$$

where $i \geq 0, n, m \in \mathbb{Z}, u_{i} \in V_{i}, \psi_{-i} \in V_{-i}, x_{n} \in V_{n}$ and $y_{m} \in V_{m}$. Let us show (3.2). We argue it by induction on $i$.

For $i=0,1$, our result follows immediately from (1.27), (1.28) and (1.29).

For $i \geq 2$. We can assume that $u_{i}=p_{i-1}\left(v_{1} \otimes u_{i-1}\right)$ for some $v_{1} \in V_{1}$ and $u_{i-1} \in V_{i-1}$ without loss of generality. Then, by the induction hypothesis, we have

$$
\begin{aligned}
B & \left(\left[p_{i-1}\left(v_{1} \otimes u_{i-1}\right), x_{n}\right], y_{m}\right) \\
& =B\left(\left[v_{1},\left[u_{i-1}, x_{n}\right]\right]-\left[u_{i-1},\left[v_{1}, x_{n}\right]\right], y_{m}\right) \\
& =B\left(\left[u_{i-1}, x_{n}\right],\left[y_{m}, v_{1}\right]\right)-B\left(\left[v_{1}, x_{n}\right],\left[y_{m}, u_{i-1}\right]\right) \\
& =B\left(x_{n},\left[\left[y_{m}, v_{1}\right], u_{i-1}\right]\right)-B\left(x_{n},\left[\left[y_{m}, u_{i-1}\right], v_{1}\right]\right) \\
& =B\left(x_{n},\left[y_{m}, p_{i-1}\left(v_{1} \otimes u_{i-1}\right)\right]\right) .
\end{aligned}
$$

Thus, we have (3.2). Similarly, we can obtain (3.3). This completes the proof.

We prove the 'universality' of Lie algebras associated with a standard quadruplet.

Proposition 3.3. Assume that a $\mathbb{Z}$-graded Lie algebra $\hat{\mathfrak{g}}=\sum_{n \in \mathbb{Z}} \hat{\mathfrak{g}}_{n}$ with a symmetric $\hat{\mathfrak{g}}$-invariant bilinear form $\hat{B}$ satisfies the following conditions:

$\hat{\mathfrak{g}}_{0}$ and $\hat{\mathfrak{g}}_{1}$ are finite-dimensional vector spaces,

the adjoint representation of $\hat{\mathfrak{g}}_{0}$ on $\hat{\mathfrak{g}}_{1}$ is completely reducible,

the restriction of $\hat{B}$ to $\hat{\mathfrak{g}}_{i} \times \hat{\mathfrak{g}}_{-i}$ is non-degenerate for each $i \geq 0$,

$\left[\hat{\mathfrak{g}}_{1}, \hat{\mathfrak{g}}_{i}\right]=\hat{\mathfrak{g}}_{i+1},\left[\hat{\mathfrak{g}}_{-1}, \hat{\mathfrak{g}}_{-j}\right]=\hat{\mathfrak{g}}_{-j-1}$ for each $i, j \geq 0$,

$\left[\hat{\mathfrak{g}}_{1}, \hat{\mathfrak{g}}_{-1}\right]=\hat{\mathfrak{g}}_{0}$.

Then $\left(\hat{\mathfrak{g}}_{0}\right.$, ad, $\left.\hat{\mathfrak{g}}_{1},\left.\hat{B}\right|_{\hat{\mathfrak{g}}_{0} \times \hat{\mathfrak{g}}_{0}}\right)$ is a standard quadruplet and the Lie algebra $L\left(\hat{\mathfrak{g}}_{0}\right.$, ad, $\hat{\mathfrak{g}}_{1}$, $\left.\left.\hat{B}\right|_{\hat{\mathfrak{g}}_{0} \times \hat{\mathfrak{g}}_{0}}\right)$ is isomorphic to $\hat{\mathfrak{g}}$ where ad stands for the adjoint representation of $\hat{\mathfrak{g}}_{0}$ on $\hat{\mathfrak{g}}_{1}$.

Obviously, $L\left(\mathfrak{g}, \rho, V, B_{0}\right)$ satisfies these conditions, i.e. $L\left(\mathfrak{g}, \rho, V, B_{0}\right)$ is, although it is defined over $\mathbb{C}$, of type $\alpha_{0}$ (see $[\mathbf{K}-\mathbf{A}]$ ). In order to prove Proposition 3.3, we prove the following lemma needed later. 
Lemma 3.4. Under the notation in Proposition 3.3, we have

$$
\left\{X_{i} \in \hat{\mathfrak{g}}_{i} \mid\left[X_{i}, Y_{-1}\right]=0 \text { for any } Y_{-1} \in \hat{\mathfrak{g}}_{-1}\right\}=\{0\}
$$

for all $i \geq 0$. In particular, a quadruplet $\left(\hat{\mathfrak{g}}_{0}\right.$, ad, $\left.\hat{\mathfrak{g}}_{1},\left.\hat{B}\right|_{\hat{\mathfrak{g}}_{0} \times \hat{\mathfrak{g}}_{0}}\right)$ satisfies (1.17).

Proof. Suppose that $X_{i} \in \hat{\mathfrak{g}}_{i}$ satisfies $\left[X_{i}, Y_{-1}\right]=0$ for any $Y_{-1} \in \hat{\mathfrak{g}}_{-1}$. Then, for any $Y_{-1} \in$ $\hat{\mathfrak{g}}_{-1}$ and $Z_{-i+1} \in \hat{\mathfrak{g}}_{-i+1}$, we have

$$
\hat{B}\left(X_{i},\left[Y_{-1}, Z_{-i+1}\right]\right)=\hat{B}\left(\left[X_{i}, Y_{-1}\right], Z_{-i+1}\right)=0 .
$$

Thus, by (3.6), (3.7) and (3.8), we obtain $X_{i}=0$.

By (3.7), ( $\hat{\mathfrak{g}}_{0}$, ad, $\left.\hat{\mathfrak{g}}_{1},\left.\hat{B}\right|_{\hat{\mathfrak{g}}_{0} \times \hat{\mathfrak{g}}_{0}}\right)$ satisfies (1.18). Hence, it is a standard quadruplet.

Let us prove Proposition 3.3. For this, we construct an isomorphism of Lie algebras from $L\left(\hat{\mathfrak{g}}_{0}\right.$, ad, $\left.\hat{\mathfrak{g}}_{1},\left.\hat{B}\right|_{\hat{\mathfrak{g}}_{0} \times \hat{\mathfrak{g}}_{0}}\right)$ to $\hat{\mathfrak{g}}$. We denote the $n$-graduation of $\left(\hat{\mathfrak{g}}_{0}\right.$, ad, $\left.\hat{\mathfrak{g}}_{1},\left.\hat{B}\right|_{\hat{\mathfrak{g}}_{0} \times \hat{\mathfrak{g}}_{0}}\right)$ by $(\hat{\mathfrak{g}})_{n}$ and the bilinear form on $L\left(\hat{\mathfrak{g}}_{0}\right.$, ad, $\left.\hat{\mathfrak{g}}_{1},\left.\hat{B}\right|_{\hat{\mathfrak{g}}_{0} \times \hat{\mathfrak{g}}_{0}}\right)$ defined in Definition 3.1 by $B$. Let $\tau_{0}$ be the identity map on $\hat{\mathfrak{g}}_{0}$ and $\tau_{1}:=\Phi^{-1}$. Then we obtain a linear isomorphism $\tau_{-1}:(\hat{\mathfrak{g}})_{-1} \rightarrow$ $\hat{\mathfrak{g}}_{-1}$ defined by $B\left(\phi_{-1}, v_{1}\right)=\hat{B}\left(\tau_{-1}\left(\phi_{-1}\right), \tau_{1}\left(v_{1}\right)\right)$ where $\phi_{-1} \in(\hat{\mathfrak{g}})_{-1}$ and $v_{1} \in(\hat{\mathfrak{g}})_{1}$. These linear isomorphisms $\tau_{i}:(\hat{\mathfrak{g}})_{i} \rightarrow \hat{\mathfrak{g}}_{i}(i=0, \pm 1)$ satisfy the following equations:

$$
\begin{aligned}
& {\left[\tau_{0}(a), \tau_{1}\left(v_{1}\right)\right]=\tau_{1}\left(\left[a, v_{1}\right]\right),} \\
& {\left[\tau_{0}(a), \tau_{-1}\left(\phi_{-1}\right)\right]=\tau_{-1}\left(\left[a, \phi_{-1}\right]\right),} \\
& {\left[\tau_{-1}\left(\phi_{-1}\right), \tau_{1}\left(v_{1}\right)\right]=\tau_{0}\left(\left[\phi_{-1}, v_{1}\right]\right),}
\end{aligned}
$$

where $a \in(\hat{\mathfrak{g}})_{0}, v_{1} \in(\hat{\mathfrak{g}})_{1}$ and $\phi_{-1} \in(\hat{\mathfrak{g}})_{-1}$. In fact, (3.9) obviously holds. Moreover, for any $b \in(\hat{\mathfrak{g}})_{0}$, we have

$$
\begin{aligned}
\hat{B}\left(\tau_{0}(b), \tau_{0}\left(\left[\phi_{-1}, v_{1}\right]\right)\right) & =B\left(b,\left[\phi_{-1}, v_{1}\right]\right) \\
& =B\left(\left[v_{1}, b\right], \phi_{-1}\right) \\
& =\hat{B}\left(\left[\tau_{1}\left(v_{1}\right), \tau_{0}(b)\right], \tau_{-1}\left(\phi_{-1}\right)\right) \\
& =\hat{B}\left(\tau_{0}(b),\left[\tau_{-1}\left(\phi_{-1}\right), \tau_{1}\left(v_{1}\right)\right]\right)
\end{aligned}
$$

and, hence, (3.11) holds. Similarly, we have (3.10).

Next, suppose that $i \geq 1$ and assume that there exist linear isomorphisms $\tau_{i-1}:(\hat{\mathfrak{g}})_{i-1} \rightarrow$ $\hat{\mathfrak{g}}_{i-1}$ and $\tau_{i}:(\hat{\mathfrak{g}})_{i} \rightarrow \hat{\mathfrak{g}}_{i}$ satisfying the following equations:

$$
\begin{aligned}
& {\left[\tau_{0}(a), \tau_{i}\left(u_{i}\right)\right]=\tau_{i}\left(\left[a, u_{i}\right]\right),} \\
& {\left[\tau_{1}\left(v_{1}\right), \tau_{i-1}\left(u_{i-1}\right)\right]=\tau_{i}\left(\left[v_{1}, u_{i-1}\right]\right),} \\
& {\left[\tau_{-1}\left(\phi_{-1}\right), \tau_{i}\left(u_{i}\right)\right]=\tau_{i-1}\left(\left[\phi_{-1}, u_{i}\right]\right),}
\end{aligned}
$$

where $a \in(\hat{\mathfrak{g}})_{0}, v_{1} \in(\hat{\mathfrak{g}})_{1}, \phi_{-1} \in(\hat{\mathfrak{g}})_{-1}, u_{i-1} \in(\hat{\mathfrak{g}})_{i-1}$ and $u_{i} \in(\hat{\mathfrak{g}})_{i}$. Then we have

$$
\tau_{i}\left(\left[\phi_{-1},\left[v_{1}, u_{i}\right]\right]\right)=\left[\tau_{-1}\left(\phi_{-1}\right),\left[\tau_{1}\left(v_{1}\right), \tau_{i}\left(u_{i}\right)\right]\right] .
$$


In fact,

$$
\begin{aligned}
\tau_{i}\left(\left[\phi_{-1},\left[v_{1}, u_{i}\right]\right]\right) & =\tau_{i}\left(\left[\left[\phi_{-1}, v_{1}\right], u_{i}\right]\right)+\tau_{i}\left(\left[v_{1},\left[\phi_{-1}, u_{i}\right]\right]\right) \\
& =\left[\tau_{0}\left(\left[\phi_{-1}, v_{1}\right]\right), \tau_{i}\left(u_{i}\right)\right]+\left[\tau_{1}\left(v_{1}\right), \tau_{i-1}\left(\left[\phi_{-1}, u_{i}\right]\right)\right] \\
& =\left[\left[\tau_{-1}\left(\phi_{-1}\right), \tau_{1}\left(v_{1}\right)\right], \tau_{i}\left(u_{i}\right)\right]+\left[\tau_{1}\left(v_{1}\right),\left[\tau_{-1}\left(\phi_{-1}\right), \tau_{i}\left(u_{i}\right)\right]\right] \\
& =\left[\tau_{-1}\left(\phi_{-1}\right),\left[\tau_{1}\left(v_{1}\right), \tau_{i}\left(u_{i}\right)\right]\right] .
\end{aligned}
$$

Thus, by Lemma 3.4, we can define a linear map $\tau_{i+1}:(\hat{\mathfrak{g}})_{i+1} \rightarrow \hat{\mathfrak{g}}_{i+1}$ by

$$
\tau_{i+1}\left(p_{i}\left(v_{1} \otimes u_{i}\right)\right):=\left[\tau_{1}\left(v_{1}\right), \tau_{i}\left(u_{i}\right)\right] .
$$

Then, by (3.7) and (3.15), we obtain that $\tau_{i+1}$ is bijective. Moreover, $\tau_{i+1}$ satisfies the following equations:

$$
\begin{aligned}
& {\left[\tau_{0}(a), \tau_{i+1}\left(u_{i+1}\right)\right]=\tau_{i+1}\left(\left[a, u_{i+1}\right]\right),} \\
& {\left[\tau_{1}\left(v_{1}\right), \tau_{i}\left(u_{i}\right)\right]=\tau_{i+1}\left(\left[v_{1}, u_{i}\right]\right),} \\
& {\left[\tau_{-1}\left(\phi_{-1}\right), \tau_{i+1}\left(u_{i+1}\right)\right]=\tau_{i}\left(\left[\phi_{-1}, u_{i+1}\right]\right),}
\end{aligned}
$$

where $a \in(\hat{\mathfrak{g}})_{0}, v_{1} \in(\hat{\mathfrak{g}})_{1}, \phi_{-1} \in(\hat{\mathfrak{g}})_{-1}, u_{i} \in(\hat{\mathfrak{g}})_{i}$ and $u_{i+1} \in(\hat{\mathfrak{g}})_{i+1}$. Indeed, (3.17) follows from a similar argument to the proof of (3.15). Equations (3.18) and (3.19) have already been shown. Thus, inductively, we obtain a linear isomorphism $\tau_{n}$ from $(\hat{\mathfrak{g}})_{n}$ to $\hat{\mathfrak{g}}_{n}$ for all $n \geq 0$. Moreover, $\tau_{n}$ induces a linear isomorphism $\tau_{-n}$ from $(\hat{\mathfrak{g}})_{-n}$ to $\hat{\mathfrak{g}}_{-n}$ which satisfies the following equation:

$$
B\left(\psi_{-n}, u_{n}\right)=\hat{B}\left(\tau_{-n}\left(\psi_{-n}\right), \tau_{n}\left(u_{n}\right)\right),
$$

where $u_{n} \in(\hat{\mathfrak{g}})_{n}$ and $\psi_{-n} \in(\hat{\mathfrak{g}})_{-n}$. Then $\tau_{-n}$ satisfies the following equations:

$$
\begin{aligned}
& {\left[\tau_{1}\left(v_{1}\right), \tau_{-n}\left(\psi_{-n}\right)\right]=\tau_{-n+1}\left(\left[v_{1}, \psi_{-n}\right]\right),} \\
& {\left[\tau_{0}(a), \tau_{-n}\left(\psi_{-n}\right)\right]=\tau_{-n}\left(\left[a, \psi_{-n}\right]\right),} \\
& {\left[\tau_{-1}\left(\phi_{-1}\right), \tau_{-n}\left(\psi_{-n}\right)\right]=\tau_{-n-1}\left(\left[\phi_{-1}, \psi_{-n}\right]\right),}
\end{aligned}
$$

where $a \in(\hat{\mathfrak{g}})_{0}, v_{1} \in(\hat{\mathfrak{g}})_{1}, \phi_{-1} \in(\hat{\mathfrak{g}})_{-1}$ and $\psi_{-n} \in(\hat{\mathfrak{g}})_{-n}$. Let us show (3.21). By (3.9), (3.11) and (3.18), for any $n \geq 0$ and $u_{n-1} \in(\hat{\mathfrak{g}})_{n-1}$, we have

$$
\begin{aligned}
\hat{B}\left(\tau_{n-1}\left(u_{n-1}\right),\left[\tau_{1}\left(v_{1}\right), \tau_{-n}\left(\psi_{-n}\right)\right]\right) & =\hat{B}\left(\left[\tau_{n-1}\left(u_{n-1}\right), \tau_{1}\left(v_{1}\right)\right], \tau_{-n}\left(\psi_{-n}\right)\right) \\
& =\hat{B}\left(\tau_{n}\left(\left[u_{n-1}, v_{1}\right]\right), \tau_{-n}\left(\psi_{-n}\right)\right) \\
& =B\left(\left[u_{n-1}, v_{1}\right], \psi_{-n}\right) \\
& =B\left(u_{n-1},\left[v_{1}, \psi_{-n}\right]\right) \\
& =\hat{B}\left(\tau_{n-1}\left(u_{n-1}\right), \tau_{-n+1}\left(\left[v_{1}, \psi_{-n}\right]\right)\right) .
\end{aligned}
$$

Hence, (3.21) holds for all $n \geq 0$. Similarly we can show (3.22) and (3.23). Summarizing the above argument, we can define a linear isomorphism $\tau$ from $L\left(\hat{\mathfrak{g}}_{0}, \operatorname{ad}, \hat{\mathfrak{g}}_{1},\left.\hat{B}\right|_{\hat{\mathfrak{g}}_{0} \times \hat{\mathfrak{g}}_{0}}\right)$ to $\hat{\mathfrak{g}}$ by

$$
\tau\left(\sum_{n \in \mathbb{Z}} x_{n}\right):=\sum_{n \in \mathbb{Z}} \tau_{n}\left(x_{n}\right),
$$


where $x_{n} \in(\hat{\mathfrak{g}})_{n}$. Then $\tau$ satisfies the equations

$$
\begin{aligned}
& {[\tau(a), \tau(x)]=\tau([a, x]),} \\
& {\left[\tau\left(v_{1}\right), \tau(x)\right]=\tau\left(\left[v_{1}, x\right]\right),} \\
& {\left[\tau\left(\phi_{-1}\right), \tau(x)\right]=\tau\left(\left[\phi_{-1}, x\right]\right),}
\end{aligned}
$$

where $a \in(\hat{\mathfrak{g}})_{0}, v_{1} \in(\hat{\mathfrak{g}})_{1}, \phi_{-1} \in(\hat{\mathfrak{g}})_{-1}$ and $x \in L\left(\hat{\mathfrak{g}}_{0}\right.$, ad, $\left.\hat{\mathfrak{g}}_{1},\left.\hat{B}\right|_{\hat{\mathfrak{g}}_{0} \times \hat{\mathfrak{g}}_{0}}\right)$. Therefore, by the definition of a Lie algebra associated with a standard quadruplet, $\tau$ is an isomorphism of Lie algebras. This completes the proof of Proposition 3.3.

\subsection{Equivalent standard quadruplets}

In this section, we introduce a notion of equivalence between standard quadruplets.

Definition 3.5. Let $\left(\mathfrak{g}^{1}, \rho^{1}, V^{1}, B_{0}^{1}\right)$ and $\left(\mathfrak{g}^{2}, \rho^{2}, V^{2}, B_{0}^{2}\right)$ be standard quadruplets. We call these quadruplets are equivalent if and only if there exists an isomorphism of Lie algebras $\sigma: \mathfrak{g}^{1} \rightarrow \mathfrak{g}^{2}$, a linear isomorphism $\tau: V^{1} \rightarrow V^{2}$ and a non-zero element $c \in \mathbb{C}$ such that

$$
\begin{aligned}
\tau\left(\rho^{1}\left(a^{1}\right) v^{1}\right) & =\rho^{2}\left(\sigma\left(a^{1}\right)\right) \tau\left(v^{1}\right), \\
B_{0}^{1}\left(a^{1}, b^{1}\right) & =c B_{0}^{2}\left(\sigma\left(a^{1}\right), \sigma\left(b^{1}\right)\right),
\end{aligned}
$$

for any $a^{1}, b^{1} \in \mathfrak{g}^{1}$ and $v^{1} \in V^{1}$. We denote this equivalence relation by

$$
\left(\mathfrak{g}^{1}, \rho^{1}, V^{1}, B_{0}^{1}\right) \simeq\left(\mathfrak{g}^{2}, \rho^{2}, V^{2}, B_{0}^{2}\right) .
$$

In particular, for a standard quadruplet $\left(\mathfrak{g}, \rho, V, B_{0}\right)$ and a non-zero element $c \in \mathbb{C}$, $\left(\mathfrak{g}, \rho, V, B_{0}\right)$ is equivalent to $\left(\mathfrak{g}, \rho, V, c B_{0}\right)$ and $\left(\mathfrak{g}, \rho_{1}, V_{1}, B_{0}\right)$ where $\left(\rho_{1}, V_{1}\right)$ is the 1 -graduation of ( $\left.\mathfrak{g}, \rho, V, B_{0}\right)$. Then we have the following proposition.

Proposition 3.6. If standard quadruplets $\left(\mathfrak{g}^{1}, \rho^{1}, V^{1}, B_{0}^{1}\right)$ and $\left(\mathfrak{g}^{2}, \rho^{2}, V^{2}, B_{0}^{2}\right)$ are equivalent, then the Lie algebras $L\left(\mathfrak{g}^{1}, \rho^{1}, V^{1}, B_{0}^{1}\right)$ and $L\left(\mathfrak{g}^{2}, \rho^{2}, V^{2}, B_{0}^{2}\right)$ are isomorphic.

Proof. By the same argument as the proof of Proposition 3.3, we can construct a linear isomorphism from $V_{n}^{1}$ to $V_{n}^{2}$ for each $n \in \mathbb{Z}$, where $V_{n}^{i}$ denotes the $n$-graduation of $\left(\mathfrak{g}^{i}, \rho^{i}, V^{i}, B_{0}^{i}\right)$ for $i=1,2$, and an isomorphism of Lie algebras from $L\left(\mathfrak{g}^{1}, \rho^{1}, V^{1}, B_{0}^{1}\right)$ to $L\left(\mathfrak{g}^{2}, \rho^{2}, V^{2}, B_{0}^{2}\right)$.

However, in general, the converse is not true. For example, let $\mathfrak{g}=\mathfrak{s l}_{4}$. Put

$$
H^{1}:=\left(\begin{array}{cccc}
1 & 0 & 0 & 0 \\
0 & 1 & 0 & 0 \\
0 & 0 & -1 & 0 \\
0 & 0 & 0 & -1
\end{array}\right), \quad H^{2}:=\left(\begin{array}{cccc}
2 & 0 & 0 & 0 \\
0 & 0 & 0 & 0 \\
0 & 0 & 0 & 0 \\
0 & 0 & 0 & -2
\end{array}\right)
$$

and

$$
\mathfrak{g}_{n}^{1}:=\left\{X \in \mathfrak{g} \mid\left[H^{1}, X\right]=2 n X\right\}, \quad \mathfrak{g}_{n}^{2}:=\left\{X \in \mathfrak{g} \mid\left[H^{2}, X\right]=2 n X\right\}
$$

for each $n \in \mathbb{Z}$. Then we obtain two gradings:

$$
\begin{aligned}
\mathfrak{g} & =\mathfrak{g}_{-1}^{1} \oplus \mathfrak{g}_{0}^{1} \oplus \mathfrak{g}_{1}^{1} \\
& =\mathfrak{g}_{-2}^{2} \oplus \mathfrak{g}_{-1}^{2} \oplus \mathfrak{g}_{0}^{2} \oplus \mathfrak{g}_{1}^{2} \oplus \mathfrak{g}_{2}^{2} .
\end{aligned}
$$


These gradings and the Killing form $K$ on $\mathfrak{g}$ satisfy the assumptions of Proposition 3.3. Therefore, by applying Proposition 3.3, it follows that the Lie algebras $L\left(\mathfrak{g}_{0}^{i}\right.$, ad, $\left.\mathfrak{g}_{1}^{i},\left.K\right|_{\mathfrak{g}_{0}^{i} \times \mathfrak{g}_{0}^{i}}\right)(i=1,2)$ are isomorphic to $\mathfrak{g}$. However, by a direct calculation, we obtain that $\mathfrak{g}_{0}^{1}$ and $\mathfrak{g}_{0}^{2}$ are isomorphic to $\mathfrak{g l}_{1} \oplus \mathfrak{s l}_{2} \oplus \mathfrak{s l}_{2}$ and $\mathfrak{g l}_{1} \oplus \mathfrak{g l}_{1} \oplus \mathfrak{s l}_{2}$, respectively (cf. [Ru-1, Ru-2, Ru-3], the theory of PVs of parabolic type). In particular, the quadruplets $\left(\mathfrak{g}_{0}^{i}, \mathrm{ad}, \mathfrak{g}_{1}^{i},\left.K\right|_{\mathfrak{g}_{0}^{i} \times \mathfrak{g}_{0}^{i}}\right)(i=1,2)$ are not equivalent.

\subsection{Loop algebras}

In this section, we give an example of a well-known infinite-dimensional Lie algebra which is isomorphic to a Lie algebra associated with a standard quadruplet.

Let $\mathfrak{g}$ be a finite-dimensional simple Lie algebra, $\mathbb{C}\left[t, t^{-1}\right]$ be the algebra of Laurent polynomials in $t$. Set $\mathcal{L}(\mathfrak{g}):=\mathbb{C}\left[t, t^{-1}\right] \otimes \mathfrak{g}$ and define the bracket product $[\cdot, \cdot]_{0}$ on $\mathcal{L}(\mathfrak{g})$ by

$$
\left[t^{n} \otimes X, t^{m} \otimes Y\right]_{0}:=t^{n+m} \otimes[X, Y]
$$

where $n, m \in \mathbb{Z}$ and $X, Y \in \mathfrak{g}$. This bracket satisfies the axioms of a Lie algebra. The infinite-dimensional Lie algebra $\mathcal{L}(\mathfrak{g})$ is called the loop algebra and we have a $\mathbb{Z}$-grading $\mathcal{L}(\mathfrak{g})=\bigoplus_{n \in \mathbb{Z}} t^{n} \otimes \mathfrak{g}$ (see [Ka, Ch. 7]).

Now, we denote by $K$ the Killing form on $\mathfrak{g}$. Then we can define a bilinear form $K_{0}$ on $\mathcal{L}(\mathfrak{g})$ by $K_{0}\left(t^{n} \otimes X, t^{m} \otimes Y\right):=\delta_{n+m, 0} K(X, Y)$, where $\delta_{n+m, 0}$ is the Kronecker delta. By applying Proposition 3.3, we have the following proposition.

PROPOSITION 3.7. Let $\mathfrak{g}$ be a finite-dimensional simple Lie algebra and $K$ the Killing form on $\mathfrak{g}$. Then the Lie algebra $L(\mathfrak{g}, \mathrm{ad}, \mathfrak{g}, K)$ is isomorphic to the loop algebra $\mathcal{L}(\mathfrak{g})$.

\subsection{A direct sum of standard quadruplets}

Definition 3.8. Let $\left(\mathfrak{g}^{1}, \rho^{1}, V^{1}, B_{0}^{1}\right)$ and $\left(\mathfrak{g}^{2}, \rho^{2}, V^{2}, B_{0}^{2}\right)$ be standard quadruplets. Let $\rho^{1} \boxplus$ $\rho^{2}$ be a representation of $\mathfrak{g}^{1} \oplus \mathfrak{g}^{2}$ on $V^{1} \oplus V^{2}$ defined by

$$
\left(\left(\rho^{1} \boxplus \rho^{2}\right)\left(a^{1}, a^{2}\right)\right)\left(v^{1}, v^{2}\right):=\left(\rho^{1}\left(a^{1}\right) v^{1}, \rho^{2}\left(a^{2}\right) v^{2}\right),
$$

where $a^{i} \in \mathfrak{g}^{i}$ and $v^{i} \in V^{i}(i=1,2)$. Let $B_{0}^{1} \oplus B_{0}^{2}$ be a bilinear form on $\mathfrak{g}^{1} \oplus \mathfrak{g}^{2}$ defined by

$$
\left(B_{0}^{1} \oplus B_{0}^{2}\right)\left(\left(a^{1}, a^{2}\right),\left(b^{1}, b^{2}\right)\right):=B_{0}^{1}\left(a^{1}, b^{1}\right)+B_{0}^{2}\left(a^{2}, b^{2}\right),
$$

where $a^{i}, b^{i} \in \mathfrak{g}^{i}(i=1,2)$. Then the quadruplet $\left(\mathfrak{g}^{1} \oplus \mathfrak{g}^{2}, \rho^{1} \boxplus \rho^{2}, V^{1} \oplus V^{2}, B_{0}^{1} \oplus B_{0}^{2}\right)$ is a standard quadruplet. We call it the direct sum of $\left(\mathfrak{g}^{1}, \rho^{1}, V^{1}, B_{0}^{1}\right)$ and $\left(\mathfrak{g}^{2}, \rho^{2}, V^{2}, B_{0}^{2}\right)$ and denote by $\left(\mathfrak{g}^{1}, \rho^{1}, V^{1}, B_{0}^{1}\right) \oplus\left(\mathfrak{g}^{2}, \rho^{2}, V^{2}, B_{0}^{2}\right)$.

By applying Proposition 3.3, we have the following proposition immediately.

Proposition 3.9. Let $\left(\mathfrak{g}^{1}, \rho^{1}, V^{1}, B_{0}^{1}\right)$ and $\left(\mathfrak{g}^{2}, \rho^{2}, V^{2}, B_{0}^{2}\right)$ be standard quadruplets. Then the Lie algebra $L\left(\left(\mathfrak{g}^{1}, \rho^{1}, V^{1}, B_{0}^{1}\right) \oplus\left(\mathfrak{g}^{2}, \rho^{2}, V^{2}, B_{0}^{2}\right)\right)$ is isomorphic to $L\left(\mathfrak{g}^{1}, \rho^{1}, V^{1}, B_{0}^{1}\right) \oplus L\left(\mathfrak{g}^{2}, \rho^{2}, V^{2}, B_{0}^{2}\right)$.

Definition 3.10. A standard quadruplet $\left(\mathfrak{g}, \rho, V, B_{0}\right)$ is said to be decomposable if and only if there exist non-trivial ideals $\mathfrak{a}$ and $\mathfrak{b}$ of $\mathfrak{g}$ and non-trivial submodules $U$ and $W$ of $V$ which 
satisfy

$$
\begin{aligned}
\mathfrak{a} \oplus \mathfrak{b} & =\mathfrak{g}, \\
U \oplus W & =V, \\
\rho(a) w & =0, \\
\rho(b) u & =0, \\
B_{0}(a, b) & =0,
\end{aligned}
$$

for any $a \in \mathfrak{a}, b \in \mathfrak{b}, u \in U$ and $w \in W$. Then $\left(\mathfrak{a},\left.\rho\right|_{\mathfrak{a}}, U,\left.B_{0}\right|_{\mathfrak{a} \times \mathfrak{a}}\right)$ and $\left(\mathfrak{b},\left.\rho\right|_{\mathfrak{b}}, W,\left.B_{0}\right|_{\mathfrak{b} \times \mathfrak{b}}\right)$ are standard quadruplets and $\left(\mathfrak{g}, \rho, V, B_{0}\right) \simeq\left(\mathfrak{a},\left.\rho\right|_{\mathfrak{a}}, U,\left.B_{0}\right|_{\mathfrak{a} \times \mathfrak{a}}\right) \oplus\left(\mathfrak{b},\left.\rho\right|_{\mathfrak{b}}, W,\left.B_{0}\right|_{\mathfrak{b} \times \mathfrak{b}}\right)$. If $\left(\mathfrak{g}, \rho, V, B_{0}\right)$ is not decomposable, it is said to be indecomposable. Any standard quadruplet can be written as a direct sum of finitely many indecomposable standard quadruplets.

Proposition 3.11. Let $\left(\mathfrak{g}, \rho, V, B_{0}\right)$ be an indecomposable standard quadruplet and $L\left(\mathfrak{g}, \rho, V, B_{0}\right)=\bigoplus_{n \in \mathbb{Z}} V_{n}$ the Lie algebra associated with it. Let $n$ be an integer such that $V_{n+1}$ is not $\{0\}$. Then for any non-zero element $x_{n} \in V_{n}$, there exists an element $v_{1} \in V_{1}$ such that $\left[v_{1}, x_{n}\right] \neq 0$.

Proof. If $n \leq 0$, we have our result immediately. Suppose that $n \geq 1$. Put

$$
U_{n}:=\left\{x_{n} \in V_{n} \mid\left[v_{1}, x_{n}\right]=0 \text { for any } v_{1} \in V_{1}\right\} .
$$

Then $U_{n}$ is a $V_{0}$-submodule of $V_{n}$. Moreover, for each $m=0, \ldots, n-1$, we can define a $V_{0}$-submodule $U_{m}$ of $V_{m}$ by

$$
U_{m}:=\left\{x_{m} \in V_{m} \mid\left[v_{1}, x_{m}\right] \in U_{m+1} \text { for any } v_{1} \in V_{1}\right\}
$$

inductively. Then we can easily show that

$$
\left[V_{-1}, U_{m}\right] \subset U_{m-1}
$$

for $m=1, \ldots, n$ inductively. Moreover, we have

$$
V_{0} \neq U_{0}
$$

In fact, since $V_{n+1} \neq\{0\}$, we can deduce that $U_{n} \neq V_{n}$. Similarly, we have $U_{m} \neq V_{m}$ for $m=0, \ldots, n-1$ inductively.

Now, since $V_{1}$ is a completely reducible $V_{0}$-module, we can take a $V_{0}$-submodule $W_{1} \subset V_{1}$ which satisfies $V_{1}=U_{1} \oplus W_{1}$. Then by the definition of $U_{0}$, we have $\left[U_{0}, W_{1}\right]=$ $\{0\}$. Put $W_{0}:=\left\{a \in V_{0} \mid B(a, b)=0\right.$ for any $\left.b \in U_{0}\right\}$. Then we have $\left[W_{0}, U_{1}\right]=\{0\}$. In fact, suppose that $a \in W_{0}$ and $u_{1} \in U_{1}$. Then for any $\phi_{-1} \in V_{-1}$, we have $\left[u_{1}, \phi_{-1}\right] \in U_{0}$ and $B\left(\left[a, u_{1}\right], \phi_{-1}\right)=B\left(a,\left[u_{1}, \phi_{-1}\right]\right)=0$, hence $\left[a, u_{1}\right]=0$. Since $\rho$ is faithful, we have $V_{0}=$ $U_{0} \oplus W_{0}$. Therefore, by the assumption that $\left(\mathfrak{g}, \rho, V, B_{0}\right)$ is an indecomposable standard quadruplet, we have $U_{0}=\{0\}$. Therefore, by Lemma 3.4 and (3.41), we can obtain $U_{m}=\{0\}$ for $m=1, \ldots, n$ by induction. In particular, $U_{n}=\{0\}$ and, thus, we have our result.

Proposition 3.12. Let $\left(\mathfrak{g}, \rho, V, B_{0}\right)$ be a standard quadruplet. If the Lie algebra $L\left(\mathfrak{g}, \rho, V, B_{0}\right)$ is finite-dimensional, $L\left(\mathfrak{g}, \rho, V, B_{0}\right)$ is semisimple. In particular, if $\left(\mathfrak{g}, \rho, V, B_{0}\right)$ is indecomposable, then $L\left(\mathfrak{g}, \rho, V, B_{0}\right)$ is simple. 
Proof. It is sufficient to show the case where $\left(\mathfrak{g}, \rho, V, B_{0}\right)$ is indecomposable. Let $\mathfrak{a}$ be a non-zero ideal of $L\left(\mathfrak{g}, \rho, V, B_{0}\right)$. Let $k$ be a positive integer such that $V_{k} \neq\{0\}$ and $V_{k+1}=\{0\}$. Then there exist integers $-k \leq n_{1}<\cdots<n_{l} \leq k$ and non-zero elements $x_{n_{1}} \in$ $V_{n_{1}}, \ldots, x_{n_{l}} \in V_{n_{l}}$ such that

$$
x:=x_{n_{1}}+\cdots+x_{n_{l}} \in \mathfrak{a} .
$$

Then by Proposition 3.11, there exist non-zero elements $v_{1}^{1}, \ldots, v_{1}^{k-n_{1}} \in V_{1}$ such that

$$
\left[v_{1}^{k-n_{1}},\left[v_{1}^{k-n_{1}-1}, \ldots,\left[v_{1}^{1}, x\right] \cdots\right]\right] \in V_{k} \backslash\{0\} .
$$

Thus, we can obtain $\mathfrak{a}_{k}:=\mathfrak{a} \cap V_{k} \neq\{0\}$ and $\mathfrak{a}_{m}:=\mathfrak{a} \cap V_{m} \neq\{0\}$ for $m=0, \ldots, k-1$ by induction. By a similar argument to the argument in the proof of Proposition 3.11, we have $\mathfrak{a}_{0}=V_{0}$, i.e. $V_{0} \subset \mathfrak{a}$. Therefore, we have $V_{1}=\left[V_{1}, V_{0}\right], V_{-1}=\left[V_{-1}, V_{0}\right] \subset \mathfrak{a}$ and thus $\mathfrak{a}=L\left(\mathfrak{g}, \rho, V, B_{0}\right)$. Therefore, $L\left(\mathfrak{g}, \rho, V, B_{0}\right)$ is simple and, thus, we have our result.

\subsection{A standard quadruplet of parabolic type}

Let $\mathfrak{g}$ be a finite-dimensional semisimple Lie algebra, $\mathfrak{h}$ a Cartan subalgebra of $\mathfrak{g}, R$ the root system of $\mathfrak{g}$ with respect to $\mathfrak{h}$. Let $K$ be the Killing form on $\mathfrak{g}$. We fix a fundamental root system $\varphi$ of $R$. Let $\theta$ be a subset of $\varphi$. Then there exists the unique element $H^{\theta} \in \mathfrak{h}$ such that $\alpha\left(H^{\theta}\right)=0$ for $\alpha \in \theta$ and $\alpha\left(H^{\theta}\right)=2$ for $\alpha \in \varphi \backslash \theta$. We call $H^{\theta}$ the grading element.

For each $n \in \mathbb{Z}$, we put $d_{n}(\theta):=\left\{X \in \mathfrak{g} \mid\left[H^{\theta}, X\right]=2 n X\right\}$ and denote $d_{0}(\theta)$ by $\mathfrak{l}_{\theta}$. Then $\mathfrak{l}_{\theta}$ is a reductive subalgebra of $\mathfrak{g}$ and acts on each $d_{n}(\theta)$ by the adjoint representation. Let $G$ be the adjoint group of $\mathfrak{g}$ and $L_{\theta}$ be the connected subgroup of $G$ corresponding to $\mathfrak{l}_{\theta}$. Then it is known that the representation of $L_{\theta}$ on $d_{1}(\theta)$ is completely reducible and $\left(L_{\theta}, d_{1}(\theta)\right)$ is a PV. Such PVs are called PVs of parabolic type (see [Ru-1, Ru-2, Ru-3]).

We can easily show that the $\mathbb{Z}$-graded Lie algebra $\mathfrak{g}=\bigoplus_{n \in \mathbb{Z}} d_{n}(\theta)$ and the Killing form $K$ on $\mathfrak{g}$ satisfy the conditions from (3.4) to (3.7). If $\left(\mathfrak{l}_{\theta}\right.$, ad, $d_{1}(\theta),\left.K\right|_{\mathfrak{l}_{\theta} \times \mathfrak{l}_{\theta}}$ ) is a standard quadruplet, i.e. $\mathfrak{l}_{\theta}=\left[d_{-1}(\theta), d_{1}(\theta)\right]$ holds, we call it a standard quadruplet of parabolic type. Then we have the following theorem.

THEOREM 3.13. Let $\left(\mathfrak{g}, \rho, V, B_{0}\right)$ be a standard quadruplet. Then the Lie algebra $L\left(\mathfrak{g}, \rho, V, B_{0}\right)$ is finite-dimensional if and only if $\left(\mathfrak{g}, \rho, V, B_{0}\right)$ is a direct sum of standard quadruplets which are equivalent to some standard quadruplet of parabolic type.

Proof. By applying Proposition 3.3, we can obtain that the Lie algebra associated to a standard quadruplet of parabolic type is finite-dimensional immediately. Let us prove the converse. We can prove it by a similar argument to the argument in [Ru-3, pp. 113-115] and to the proof of Lemma 1.63 of [Wa]. Assume that $L\left(\mathfrak{g}, \rho, V, B_{0}\right)=\bigoplus_{n \in \mathbb{Z}} V_{n}$ is finitedimensional. Without loss of generality, we can assume that $\left(\mathfrak{g}, \rho, V, B_{0}\right)$ is indecomposable. Then, by Proposition $3.12, L\left(\mathfrak{g}, \rho, V, B_{0}\right)$ is simple. Hence, the bilinear form $B$ defined in Definition 3.1 is a scalar multiple of the Killing form $K_{L}$ on $L\left(\mathfrak{g}, \rho, V, B_{0}\right)$, i.e. there exists a non-zero element $c \in \mathbb{C}$ such that $K_{L}=c B$ (see [Wa, Theorem 1.31]). Moreover, by the theory of Lie algebras, a derivation $\delta$ on $L\left(\mathfrak{g}, \rho, V, B_{0}\right)$ defined by $\delta\left(\sum_{n \in \mathbb{Z}} x_{n}\right):=\sum_{n \in \mathbb{Z}} 2 n x_{n}$, where $x_{n} \in V_{n}$, is an inner derivation, i.e. there exists an element $H \in L\left(\mathfrak{g}, \rho, V, B_{0}\right)$ such that $\delta=\operatorname{ad}(H)$. Since $\operatorname{ad}(H)$ is diagonalizable, there exists a Cartan 
subalgebra $\mathfrak{h}$ of $L\left(\mathfrak{g}, \rho, V, B_{0}\right)$ such that $H \in \mathfrak{h}$. Let $R$ be the root system of $L\left(\mathfrak{g}, \rho, V, B_{0}\right)$ with respect to $\mathfrak{h}$. Let us construct a fundamental system of $R$.

Suppose that $\left[V_{0}, V_{0}\right] \neq\{0\}$. By the definition of $H, \mathfrak{h}$ is also a Cartan subalgebra of $V_{0}$. Put $\overline{\mathfrak{h}}:=\mathfrak{h} \cap\left[V_{0}, V_{0}\right]$ and denote the restriction of $\gamma \in R$ to $\overline{\mathfrak{h}}$ by $\bar{\gamma}$. Then there exists a subset $\theta=\left\{\alpha^{1}, \ldots, \alpha^{r}\right\}$ of $R$ such that $\left\{\overline{\alpha^{1}}, \ldots, \overline{\alpha^{r}}\right\}$ is a fundamental root system of the root system of $\left[V_{0}, V_{0}\right]$ with respect to $\overline{\mathfrak{h}}$. Put

$$
\begin{aligned}
\varphi^{\prime}: & =\left\{\beta \in R \mid \beta(H)=2, \beta-\alpha^{s} \notin R \text { for } s=1, \ldots, r\right\} \\
& =\left\{\beta^{1}, \ldots, \beta^{l}\right\} .
\end{aligned}
$$

Then $\theta \cup \varphi^{\prime}=\left\{\alpha^{1}, \ldots, \alpha^{r}, \beta^{1}, \ldots, \beta^{l}\right\}$ is a fundamental root system of $R$. In fact, we can easily show that any root can be written as $n(1) \alpha^{1}+\cdots+n(r) \alpha^{r}+m(1) \beta^{1}+\cdots+m(l) \beta^{l}$ where all $n(i)$ and $m(j)$ are non-negative integers or all $n(i)$ and $m(j)$ are non-positive integers. Thus, it is enough to show that $\theta \cup \varphi^{\prime}$ is linearly independent over $\mathbb{R}$. Suppose that $c(1), \ldots, c(r), d(1), \ldots, d(l) \in \mathbb{R}$ satisfy

$$
c(1) \alpha^{1}+\cdots+c(r) \alpha^{r}+d(1) \beta^{1}+\cdots+d(l) \beta^{l}=0 .
$$

By renumbering the elements of $\theta \cup \varphi^{\prime}$, we can suppose that

$$
\begin{aligned}
& c(1), \ldots, c(s) \geq 0, \quad c(s+1), \ldots, c(r)<0, \\
& d(1), \ldots, d(t) \geq 0, \quad d(t+1), \ldots, d(l)<0
\end{aligned}
$$

without loss of generality. Put

$$
\begin{aligned}
\lambda & :=c(1) \alpha^{1}+\cdots+c(s) \alpha^{s}+d(1) \beta^{1}+\cdots+d(t) \beta^{t} \\
& =-c(s+1) \alpha^{s+1}-\cdots-c(r) \alpha^{r}-d(t+1) \beta^{t+1}-\cdots-d(l) \beta^{l} .
\end{aligned}
$$

Then we have

$$
\begin{aligned}
0 \leq & K_{L}\left(H_{\lambda}, H_{\lambda}\right) \\
= & -\sum_{1 \leq i \leq s, s+1 \leq j \leq r} c(i) c(j) K_{L}\left(H_{\alpha^{i}}, H_{\alpha^{j}}\right)-\sum_{1 \leq i \leq t, t+1 \leq j \leq l} d(i) d(j) K_{L}\left(H_{\beta^{i}}, H_{\beta^{j}}\right) \\
& -\sum_{1 \leq i \leq s, t+1 \leq j \leq l} c(i) d(j) K_{L}\left(H_{\alpha^{i}}, H_{\beta^{j}}\right)-\sum_{1 \leq i \leq t, s+1 \leq j \leq r} d(i) c(j) K_{L}\left(H_{\beta^{i}}, H_{\alpha^{j}}\right),
\end{aligned}
$$

where $H_{\gamma}$ denotes the element in $\mathfrak{h}$ corresponding to $\gamma \in \mathfrak{h}^{*}$ which satisfies $K_{L}\left(H_{\gamma}, h\right)=$ $\gamma(h)$ for any $h \in \mathfrak{h}$. By the definition of $\theta \cup \varphi^{\prime}$, it follows that $\gamma^{1}-\gamma^{2} \notin R$ for any $\gamma^{1}, \gamma^{2} \in$ $\theta \cup \varphi^{\prime}$ and, thus, $K_{L}\left(H_{\gamma^{1}}, H_{\gamma^{2}}\right) \leq 0\left(\gamma^{1} \neq \gamma^{2}\right)$. Therefore, we have $K_{L}\left(H_{\lambda}, H_{\lambda}\right)=0$ and $\lambda=0$. Therefore, we have

$$
\begin{aligned}
& c(1) \alpha^{1}(H)+\cdots+c(s) \alpha^{s}(H)+d(1) \beta^{1}(H)+\cdots+d(t) \beta^{t}(H) \\
& \quad=2 d(1)+\cdots+2 d(t)=0
\end{aligned}
$$

and thus $d(1)=\cdots=d(t)=0$. Moreover, it follows that $c(1)=\cdots=c(s)=0$ from the assumption that $\theta$ is linearly independent. Similarly, we have $c(s+1)=\cdots=c(r)=$ $d(t+1)=\cdots=d(l)=0$. Therefore, $\theta \cup \varphi^{\prime}$ is a fundamental root system of $R$. 
If $\left[V_{0}, V_{0}\right]=\{0\}$, i.e. $V_{0}$ is commutative, then a set $\varphi:=\{\beta \in R \mid \beta(H)=2\}$ is a fundamental root system of $R$. Indeed, we can show it by a similar argument.

Summarizing the above argument, it follows that $\left(\mathfrak{g}, \rho_{1}, V_{1}, c B_{0}\right)$ is a standard quadruplet of parabolic type and $\left(\mathfrak{g}, \rho, V, B_{0}\right)$ is equivalent to it.

It follows from Theorem 3.13 that any semisimple Lie algebra can be obtained from some standard quadruplet. In particular, for a positive integer $m \geq 2$, we can obtain $\mathfrak{s l}_{m+1}$ and $\mathfrak{s o}_{2 m+1}$ from standard quadruplets given in Examples 1.14 and 1.15, respectively.

\section{REFERENCES}

[Bu] N. Bourbaki. Lie Groups and Lie Algebra. Springer, Berlin, 1989.

[Ka] V. G. Kac. Infinite Dimensional Lie Algebras, 3rd edn. Cambridge University Press, Cambridge, 1990.

[K-A] S. Kaneyuki and H. Asano. Graded Lie algebras and generalized Jordan triple systems. Nagoya Math. J. 112 (1988), 81-115.

[Ru-1] H. Rubenthaler. Espaces préhomogènes de type parabolique. Lect. Math. Kyoto Univ. 14 (1982), 189-221.

[Ru-2] H. Rubenthaler. Espaces préhomogènes de type parabolique. Thèse d'Etat, Université de Strasbourg, 1982.

[Ru-3] H. Rubenthaler. Algèbres de Lie et espaces préhomogènes (Travaux en cours). Hermann, Paris, 1992.

[S-K] M. Sato and T. Kimura. A classification of irreducible prehomogeneous vector spaces and their relative invariants. Nagoya Math. J. 65 (1977), 1-155.

[Wa] M. Wakimoto. Infinite-Dimensional Lie Algebras. American Mathematical Society, Providence, RI, 2001.

Nagatoshi Sasano

Department of Mathematics

Kyushu University

Nishi-ku, Fukuoka 819-0395

Japan

(E-mail:n-sasano@math.kyushu-u.ac.jp) 